\title{
Versatile and accurate schemes of discretization in the scattering analysis of 2D composite objects with penetrable or perfectly conducting regions
}

\author{
Ivan Sekulic, Eduard Ubeda and Juan M. Rius
}

\begin{abstract}
The Method-of-Moment (MoM) discretization of boundary integral equations in the scattering analysis of closed infinitely long (2D) objects, perfectly conducting or penetrable, is traditionally carried out with continuous piecewise linear basis functions, which embrace pairs of adjacent segments. This is numerically advantageous because the discretization of the transversal component of the scattered fields, electric (TE) or magnetic (TM), becomes free from hypersingular Kernel contributions. In the analysis of composite objects, though, the imposition of the continuity requirement around junction nodes, where the boundaries of several regions intersect, becomes especially awkward. In this paper, we present, for the scattering analysis of composite objects, a new combined discretization of the Poggio-Miller-Chang-Harrington-Wu-Tsai (PMCHWT) integral equation, for homogeneous dielectric regions, and the Electric-Field Integral Equation (EFIE), for perfectly conducting regions, such that the basis functions are defined strictly on each segment, with no continuity constraint between adjacent segments. We show the improved observed accuracy with the proposed TE-PMCHWT implementation on several dielectric objects with sharp edges and corners and moderate or high contrasts. Furthermore, we illustrate the versatility of these schemes in the analysis of $2 \mathrm{D}$ composite piecewise homogeneous objects without sacrificing accuracy with respect to the conventional implementations.
\end{abstract}

Index Terms-Method of Moments, Integral equations, composite objects, Electric-Field Integral Equation, PMCHWT formulation

\section{INTRODUCTION}

The discretization by the Method of Moments (MoM) [1] and Galerkin testing of, respectively, the Electric-Field Integral Equation (EFIE) [2], for single perfectly conducting (PEC) objects, or the Poggio-Miller-Chang-Harrington-Wu-Tsai (PMCHWT) formulation [3]-[5], for single penetrable objects, is traditionally based on divergence-conforming functions, e.g. RWG [2][6], so that normal continuity is preserved across edges. In the particular case of infinitely long (2D) bodies, these implementations are normally carried out with continuous piecewise linear basis functions [7][8]. These are edge-based strategies (node-based in 2D) because the basis functions embrace two adjacent cells in the meshed boundary. Moreover, these schemes are conforming, hence with converging solutions [9][10], because the finite dimensional subspace in the current expansion lies in the physical function space [11] and the testing functions span the dual of the range of the operators [12]-[14]. The divergence-conforming choice is especially advantageous for the computation of the impedance matrix because of the cancellation of the hypersingular Kernel contributions. In practice, these schemes can be extended naturally to the scattering analysis of composite objects, made up of different homogeneous regions, PEC or penetrable, as long as there are no junctions, such as, for example, coated metallic objects or multilayered penetrable structures [15][16]. Junctions stand for boundary lines where more than two regions intersect [17]-[19]. In general, for an arbitrary composite object, they are modeled with edges (nodes in 2D).

However, the development of conforming schemes for composite objects with junctions becomes particularly cumbersome because continuity conditions need to be applied at junctions through specially tailored functions [18][19]. The conventional edge-based MoM-codes are then modified accordingly by inserting exceptions at junctions. This is a somewhat convoluted task that involves the search for junctions, the identification of the regions intersecting at each junction and the choice of the appropriate continuity condition (metallic-penetrable or penetrable). In practice, it is common to simplify such analysis by picturing the composite object as a set of disjoint objects, corresponding to each of the regions, immersed in a host medium, with separation distances tending to zero. Nevertheless, the amount of unknowns gets increased because redundant unknowns need to be defined [20].

In this paper, we present a new, robust and accurate method for the scattering analysis of 2D piecewise homogeneous objects with junctions, based on the discretization of the EFIE, over boundaries enclosing PEC regions, and of the PMCHWT formulation, over interface boundaries between different penetrable regions. We propose the expansion of the electric and magnetic currents with piecewise linear basis functions, discontinuous at nodes. The resulting EFIE-PMCHWT implementation gives rise to hypersingular Kernel contributions, which we evaluate numerically by testing the electric - or magnetic - field equations, in the Transversal Electric (TE) - or Transversal Magnetic (TM) implementations over domains off the boundary segmentation, inside the region where, in light of the surface equivalence theorem, the fields must be zero. Whereas the surface scheme [21] tests the fields over a set of trapezoids attached to the boundary, the tangential-normal scheme [22] makes use of a set of pairs of adjacent segments, such that one matches a boundary segment and the other one is normally oriented.

These discretization strategies are nonconforming because the subspaces of electric and magnetic currents, with no imposed continuity transition between adjacent cells, do not belong to the function spaces of currents. In the scattering analysis of sharp-edged conductors, nonconforming 
MoM-implementations of the (TE-)EFIE, based on the discontinuous cell-based expansion of the current monopolar-RWG (3D) or discontinuous piecewise linear (2D) - exhibit faster converging solutions than the conventional conforming schemes [22]-[25]. On the other hand, the point-based Locally Corrected Nyström (LCN) method, another nonconforming scheme with discontinuous current expansion [26]-[29], shows for low-order expansions less accurate solutions than continuous implementations for sharp-edged conductors [27][30]. In any case, these schemes avoid the preliminary task of edge (or junction) search required for the conforming schemes and allow the management of nonconformal meshes, with some nonmatching edges between adjacent facets [31][32]. Although the edge-search effort is small when compared with the matrix generation and the solution of the system, this computational load becomes increasingly evident as the meshes involve more facets.

In this paper, we show with Radar Cross Section (RCS) and near-field results that our nonconforming discretization of the TE-PMCHWT provides improved accuracy, versus the number of unknowns, in the analysis of single sharp-edged dielectric objects with moderate or high contrasts. Furthermore, we show that these EFIE-PMCHWT implementations, with segment-based expansion and testing schemes, excel as flexible and versatile tools in the analysis of composite objects when compared with the conventional node-based implementations. Although our nonconforming EFIE-PMCHWT implementation relies on the elaborate generation of the impedance elements, the burdensome management of junctions of the conforming schemes is circumvented because only segment-to-segment interactions are considered. Especially, since the basis functions of our nonconforming schemes do not span multiple regions, they become well suited to enhance the Domain Decomposition Methods [33] for the agile analysis of big composite structures resulting from the juxtaposition of independent meshed subdomains.

\section{THEORETICAL BACKGROUND}

Field singularities arising in infinitely long dielectric or magnetic sharp wedges, with aperture angle $\alpha$, are associated with the transverse component of, respectively, the electric or magnetic fields [34]. Singular field performance near penetrable wedges, sharp $\left(\alpha<180^{\circ}\right)$ or reentrant $\left(\alpha>180^{\circ}\right)$, occurs whenever the transverse field component of the impinging plane wave is, respectively, parallel or perpendicular, to the line bisecting the wedge [34]. In the TE (or TM) scattering analysis of dielectric (or magnetic) sharp wedges, the singularity of the magnetic (or electric) current and the electric (or magnetic) charge density near the wedge becomes more pronounced as the relative permittivity (or permeability) rises (see [Table 4.2, 34] and [Fig. 4.28, 34]).

As we show in this paper, our nonconforming discretization of the TE-PMCHWT formulation for dielectric sharp wedges, or, similarly, of the TM-PMCHWT formulation for magnetic sharp wedges [35], must provide a better capture of the singular electric-field or magnetic-field performance, respectively, than the conventional piecewise continuous scheme because improved accuracy, versus the number of unknowns, is observed. Conversely, the analogous implementations of the TM-PMCHWT for dielectric wedges - or of TE-PMCHWT for magnetic wedges - do not provide improved accuracy, versus the number of unknowns, because, consistently, in these cases field singularities do not occur [34]. In any case, all these nonconforming PMCHWT implementations, segment-based, are very useful for the agile management of junctions in composite objects.

Meixner and others characterized the specific behavior of electromagnetic fields near sharp edges [34]-[38]. Several basis functions incorporate the Meixner subsets for the rigorous capture of the singular behavior of the current near edges in perfectly conducting sharp-wedges [39]-[42], where the singular coefficients of the singular terms are obtained from the aperture angle of the wedge. The definition of the subsets for dielectric wedges, though, requires the numerical solution of transcendental equations that depend on the aperture angle and the dielectric contrast [34][38]. The use of our discontinuous piecewise linear schemes in these cases appears as a robust option because, unlike the elaborate singular-field implementations with the Meixner subsets, our approach does not require the a priori knowledge of aperture angles or the dielectric contrasts of the body. In this paper, we introduce our nonconforming schemes in the context of the scattering analysis of 2D dielectric objects because the well-established emergence of the singular field-performance in the TE scattering of dielectric infinitely long sharp wedges with moderate or high dielectric contrasts [34] can be associated in a straightforward manner with the observed improved performance of our discontinuous piecewise linear TE-PMCHWT implementations.

\section{NONCONFORMING TE-EFIE FOR CONDUCTORS}

For a z-infinite perfectly conducting closed body illuminated by a TE plane wave, the expansion of the electric current $\boldsymbol{J}$ with a set of discontinuous piecewise linear basis functions gives rise to

$$
\boldsymbol{J} \simeq \sum_{p=1}^{2} \sum_{n=1}^{N s} J_{n}^{p} \boldsymbol{f}_{n}^{p}
$$

where $N s$ denotes the number of segments over the transversal boundary of the body. The sequences $\left\{J_{n}^{1}\right\},\left\{J_{n}^{2}\right\}$ denote the unknown coefficients in the expansion of the electric current. The sets of discontinuous basis functions $\left\{\boldsymbol{f}_{1}^{1} \ldots \boldsymbol{f}_{N s}^{1}, \boldsymbol{f}_{1}^{2} \ldots \boldsymbol{f}_{N s}^{2}\right\}$ are defined as

$$
\boldsymbol{f}_{n}^{p}\left(\boldsymbol{\rho}^{\prime}\right)=\frac{1}{h_{n}}\left(\boldsymbol{\rho}^{\prime}-\boldsymbol{\rho}_{n}^{p}\right) \quad \boldsymbol{\rho}^{\prime} \in L_{n} \quad p=1,2
$$

where $L_{n}$ stands for the $n$th segment arising from the boundary segmentation, with length $h_{n}$, and $\rho_{n}^{1}, \rho_{n}^{2}$ represent the position vectors of the endpoints of $L_{n}$ (see Fig. 1).

The approximation of the TE scattered electric field from the expansion of $\boldsymbol{J}$ in (1), yields 


$$
\boldsymbol{E}^{S}(\boldsymbol{\rho}) \simeq-j k \eta \sum_{p=1}^{2} \sum_{n=1}^{N s} J_{n}^{p} \boldsymbol{A}_{n}^{p}(k, \boldsymbol{\rho})-\sum_{p=1}^{2} \sum_{n=1}^{N s} J_{n}^{p} \nabla \Phi_{n}^{p}(k, \boldsymbol{\rho})
$$

where $k$ and $\eta$ denote, respectively, the wavenumber and the impedance of the host medium, where the object is immersed. The two source contributions $(p=1,2)$ at the $n$th segment in the discretization of the potentials $\Phi_{n}^{p}$ and $\boldsymbol{A}_{n}^{p}$ yield $[21][22]$

$$
\begin{gathered}
\boldsymbol{A}_{n}^{p}(k, \boldsymbol{\rho})=\int_{L_{n}} G_{k}\left(\boldsymbol{\rho}, \boldsymbol{\rho}^{\prime}\right) \boldsymbol{f}_{n}^{p}\left(\boldsymbol{\rho}^{\prime}\right) d l^{\prime} \\
\Phi_{n}^{p}(k, \boldsymbol{\rho})=j \frac{\eta}{k}\left[\frac{1}{h_{n}} \int_{L_{n}} G_{k}\left(\boldsymbol{\rho}, \boldsymbol{\rho}^{\prime}\right) d l^{\prime}-G_{k}\left(\boldsymbol{\rho}, \boldsymbol{\rho}_{n}^{p+1}\right)\right]
\end{gathered}
$$

where the superscript in (5) assumes modulo two arithmetic. The Green's function $G_{k}$ of the host medium is defined as

$$
G_{k}\left(\boldsymbol{\rho}, \boldsymbol{\rho}^{\prime}\right)=\frac{1}{4 j} H_{0}^{(2)}(k R), \quad R=\left|\boldsymbol{\rho}-\boldsymbol{\rho}^{\prime}\right|
$$

where $k=\omega / \sqrt{\mu \varepsilon} ; \eta=\sqrt{\mu / \varepsilon}$ and $\omega$ denotes the angular frequency in the $e^{j o t}$ time-harmonic dependence assumed for fields and currents. The constants $\varepsilon, \mu$ denote the permittivity and the permeability, respectively, of the host medium.

The Galerkin testing of the gradient of the electric scalar potential $\nabla \Phi_{n}^{p}$ in (3) cannot be evaluated numerically for self or node-adjacent interactions because of the hypersingular contributions in $\nabla G_{k}\left(\boldsymbol{\rho}, \boldsymbol{\rho}_{n}^{p+1}\right)$, behaving as $O\left(\left|\boldsymbol{\rho}-\boldsymbol{\rho}_{n}^{p+1}\right|^{-1}\right)$ when the field point approaches the endpoints of the segment. In this paper, we present two non-Galerkin testing schemes that ensure a proper numerical evaluation of such contributions by testing the electric fields inside the body under analysis, off the boundary line. These testing functions are devised to have good coupling with the electric fields generated by the discontinuous piecewise linear basis functions.

\section{A. Surface testing}

The surface testing is carried out over a set of quadrilaterals $\left\{S_{m}\right\}$ attached to the set of segments over the boundary $\left\{L_{m}\right\}$, inside the region where the fields are zero (Fig. 1). The quadrilaterals can be defined either conformal or nonconformal to the boundary. Whereas the former establishes trapezoids with two sides parallel to the matching segments and with two sides bisecting the angles formed by the adjacent segments, the latter adopts rectangles. This definition appears more flexible because no a priori knowledge of the boundary shape is required. For segments with a node lying on an abrupt sharp edge, though, the testing rectangle may break out of the boundary interface and some numerical error may appear.
We define the set of surface testing functions $\left\{\boldsymbol{P}_{1}^{1} \ldots \boldsymbol{P}_{N s}^{1}, \boldsymbol{P}_{1}^{2} \ldots \boldsymbol{P}_{N s}^{2}\right\}$ as

$$
\begin{aligned}
\boldsymbol{P}_{m}^{q}(\boldsymbol{\rho})= & \frac{1}{H_{m} h_{m}}\left[\left(\boldsymbol{\rho}-\boldsymbol{\rho}_{m}^{q}\right) \cdot \hat{l}_{m}\right] \hat{l}_{m} \\
& +(-1)^{q} \frac{1}{H_{m} h_{m}}\left[\left(\boldsymbol{\rho}_{m}^{q}-\boldsymbol{\rho}\right) \cdot \hat{n}_{m}\right] \tan \alpha_{m}^{q} \cdot \hat{l}_{m} \\
& \boldsymbol{\rho} \in S_{m} \quad 1 \leq m \leq N s \quad q=1,2
\end{aligned}
$$

where $H_{m}$ denotes the height of the $m$ th quadrilateral and $\hat{n}_{m}$, $\hat{l}_{m}$ represent, respectively, the unit normal and tangential vectors to the $m$ th segment. The parameters $\alpha_{m}^{1}, \alpha_{m}^{2}$ denote the angles of the sides of the quadrilaterals meeting the endpoints of the $m$ th segment, respectively, $\boldsymbol{\rho}_{m}^{1}, \boldsymbol{\rho}_{m}^{2}$, with respect to the normal direction to the segment. For testing elements that are nonconformal to the boundary, these sides are perpendicular to the segment, whereby $\alpha_{m}^{1}=\alpha_{m}^{2}=0$ and the second term in (7) gets cancelled.

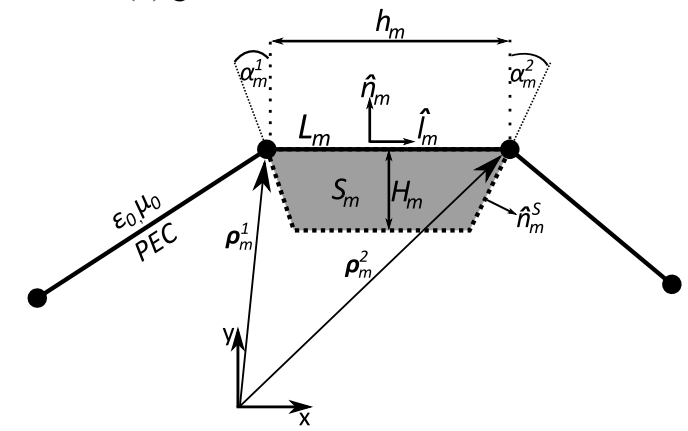

Fig. 1. $m$ th segment arising from the discretized section of an infinitely long perfectly conducting cylinder where the surface testing scheme is defined conformal to the boundary.

The surface tested nonconforming discretization of the TE-EFIE results in the following matrix system

$$
E_{i n c}^{\mathbf{S},[m, q]}=\sum_{n=1}^{N s} Z_{[n, 1]}^{\mathbf{S},[m, q]} J_{n}^{1}+\sum_{n=1}^{N s} Z_{[n, 2]}^{\mathbf{S},[m, q]} J_{n}^{2} \quad q=1,2 \quad 1 \leq m \leq N S
$$

where the excitation vector stands for

$$
E_{i n c}^{\mathrm{S},[m, q]}=\iint_{S_{m}} \boldsymbol{P}_{m}^{q}(\boldsymbol{\rho}) \cdot \boldsymbol{E}_{i n c}(\boldsymbol{\rho}) d S
$$

and $\boldsymbol{E}_{\text {inc }}(\boldsymbol{\rho})$ denotes the TE-incident electric field. The definition of the impedance elements in (8) yields

$$
\begin{aligned}
Z_{[n, p]}^{\mathrm{S},[m, q]}= & j k \eta \iint_{S_{m}} \boldsymbol{P}_{m}^{q}(\boldsymbol{\rho}) \cdot \boldsymbol{A}_{n}^{p}(k, \boldsymbol{\rho}) d S \\
& +\iint_{S_{m}} \boldsymbol{P}_{m}^{q}(\boldsymbol{\rho}) \cdot \nabla \Phi_{n}^{p}(k, \boldsymbol{\rho}) d S
\end{aligned}
$$

where, for self or node-adjacent interactions, the second surface integral at the right-hand side can manage the singular 
terms in $\nabla \Phi_{n}^{p}$ because an extra degree of freedom is incorporated with respect to the Galerkin scheme. In our implementation, we compute (10), equivalently, as

$$
\begin{aligned}
& Z_{[n, p]}^{\mathrm{S},[m, q]}=j k \eta \iint_{S_{m}} \boldsymbol{P}_{m}^{q}(\boldsymbol{\rho}) \cdot \boldsymbol{A}_{n}^{p}(k, \boldsymbol{\rho}) d S \\
& \quad+\int_{\partial S_{m}} \Phi_{n}^{p}(k, \boldsymbol{\rho}) \boldsymbol{P}_{m}^{q}(\boldsymbol{\rho}) \cdot \hat{n}_{m}^{\mathbf{S}} d l-\iint_{S_{m}} \nabla \cdot \boldsymbol{P}_{m}^{q}(\boldsymbol{\rho}) \Phi_{n}^{p}(k, \boldsymbol{\rho}) d S
\end{aligned}
$$

where $\hat{n}_{m}^{\mathrm{s}}$ stands for the unit vector normal to the boundary of the rectangular domain $S_{m}$ and pointing outwards.

\section{B. Tangential-normal testing}

The tangential-normal testing is defined over pairs of segments sharing a node arising from the segmentation of the boundary line. Whereas one testing segment matches a cell arising from the boundary segmentation, the other segment is oriented towards the region where the fields, in light of the equivalence principle, are zero (Fig. 2). The tangential-normal testing scheme can also be defined conformal or nonconformal to the boundary depending whether the off-boundary segment is aligned over the direction bisecting the angle between adjacent segments or perpendicular to the matching segment.

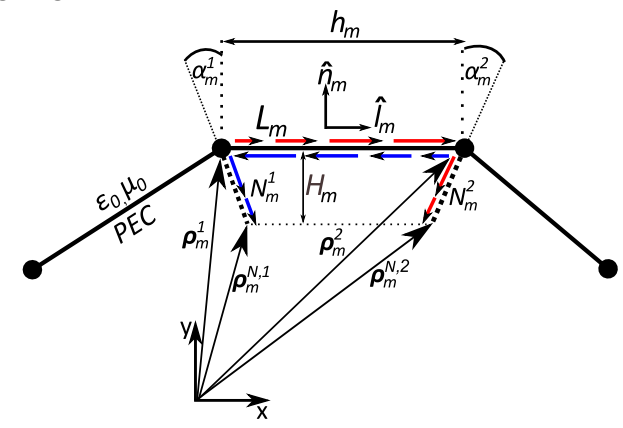

Fig. 2. $m$ th segment arising from the discretized section of an infinitely long perfectly conducting cylinder where the tangential-normal testing scheme is defined conformal to the boundary.

The definition, with modulo two arithmetic, of the tangential-normal testing functions, $\left\{\boldsymbol{t}_{1}^{1} \ldots \boldsymbol{t}_{N s}^{1}, \boldsymbol{t}_{1}^{2} \ldots \boldsymbol{t}_{N s}^{2}\right\}$ yields

$$
\begin{aligned}
& \boldsymbol{t}_{m}^{q}(\boldsymbol{\rho})= \begin{cases}\frac{1}{h_{m}}\left(\boldsymbol{\rho}-\boldsymbol{\rho}_{m}^{q}\right) & \boldsymbol{\rho} \in L_{m} \\
\frac{1}{h_{m}^{N, q+1}}\left(\boldsymbol{\rho}_{m}^{N, q+1}-\boldsymbol{\rho}\right) & \boldsymbol{\rho} \in N_{m}^{q+1}\end{cases} \\
& q=1,2 \quad 1 \leq m \leq N s
\end{aligned}
$$

where $\boldsymbol{t}_{m}^{1}$ and $\boldsymbol{t}_{m}^{2}$ represent the two tangential-normal functions sharing the $m$ th segment $L_{m}$ and $N_{m}^{1}, N_{m}^{2}$ denote two segments with lengths $h_{m}^{N, 1}$ and $h_{m}^{N, 2}$ evolving inwards from the endpoints of $L_{m}$, respectively, $\boldsymbol{\rho}_{m}^{1}$ and $\boldsymbol{\rho}_{m}^{2}$, to $\boldsymbol{\rho}_{m}^{N, 1}, \boldsymbol{\rho}_{m}^{N, 2}$ , inside the null-field region (see Fig. 2).
The discretization of the TE-EFIE with discontinuous piecewise linear basis functions and tangential-normal testing leads to the following matrix system

$$
E_{i n c}^{\mathbf{T},[m, q]}=\sum_{n=1}^{N s} Z_{[n, 1]}^{\mathbf{T},[m, q]} J_{n}^{1}+\sum_{n=1}^{N s} Z_{[n, 2]}^{\mathbf{T},[m, q]} J_{n}^{2} \quad q=1,2 \quad 1 \leq m \leq N S
$$

where the excitation vector yields

$$
E_{i n c}^{\mathbf{T},[m, q]}=\int_{L_{m} \cup N_{m}^{q+1}} \boldsymbol{t}_{m}^{q}(\boldsymbol{\rho}) \cdot \boldsymbol{E}_{i n c}(\boldsymbol{\rho}) d l
$$

and the impedance elements are defined as (see Fig. 2)

$$
\begin{aligned}
Z_{[n, p]}^{\mathbf{T},[m, q]}= & j k \eta \int_{L_{m} \cup N_{m}^{q+1}} \boldsymbol{t}_{m}^{q}(\boldsymbol{\rho}) \cdot \boldsymbol{A}_{n}^{p}(k, \boldsymbol{\rho}) d l \\
& +\int_{L_{m} \cup N_{m}^{q+1}} \boldsymbol{t}_{m}^{q}(\boldsymbol{\rho}) \cdot \nabla \Phi_{n}^{p}(k, \boldsymbol{\rho}) d l
\end{aligned}
$$

In view of the continuity along $L_{m} \cup N_{m}^{1}$ and $L_{m} \cup N_{m}^{2}$ of, respectively, $\boldsymbol{t}_{m}^{2}$ and $\boldsymbol{t}_{m}^{1}$, (15) can be simplified into

$$
\begin{aligned}
Z_{[n, p]}^{\mathrm{T},[m, q]} & =j k \eta \int_{L_{m} \cup N_{m}^{q+1}} \boldsymbol{t}_{m}^{q}(\boldsymbol{\rho}) \cdot \boldsymbol{A}_{n}^{p}(k, \boldsymbol{\rho}) d l \\
& -\int_{L_{m} \cup N_{m}^{q+1}} \nabla \cdot \boldsymbol{t}_{m}^{q}(\boldsymbol{\rho}) \Phi_{n}^{p}(k, \boldsymbol{\rho}) d l
\end{aligned}
$$

where the second line integral can now handle the $\log \left(\left|\boldsymbol{\rho}-\boldsymbol{\rho}_{n}^{p+1}\right|\right)$-singular contributions in $G_{k}\left(\boldsymbol{\rho}, \boldsymbol{\rho}_{n}^{p+1}\right)$. Interestingly, the generation of the impedance element in (16) demands less computational burden than in (11), for the surface tested approach.

\section{NONCONFORMING PMCHWT FOR SINGLE DIELECTRICS}

In general, the scattering analysis of a homogeneous z-infinite penetrable body, with arbitrary section, illuminated by a TE plane wave is carried out, in light of the equivalence theorem, through the superposition of the scattered fields, $\boldsymbol{E}_{i}^{S}, \boldsymbol{H}_{i}^{S}$, associated with the homogeneous problems of, respectively, regions $i=1$ and $i=2$ (see Fig. 3). These fields are generated by the currents over the dielectric interface, $\boldsymbol{J}_{i}, \boldsymbol{M}_{i}$ so that the total fields arising from the summation of incident and scattered fields, $\boldsymbol{E}_{i}, \boldsymbol{H}_{i}$ are zero outside respective regions (see Fig. 3).

We assume that the incident electric and magnetic fields in the original problem are in region 1 , whereby the total fields, electric or magnetic, generated in the homogeneous problems associated with region $i, \boldsymbol{C}_{i}$, become

$$
\boldsymbol{C}_{1}(\boldsymbol{\rho})=\left\{\begin{array}{cl}
\boldsymbol{C}_{1}^{S}(\boldsymbol{\rho})+\boldsymbol{C}_{i n c}(\boldsymbol{\rho}) & \boldsymbol{\rho} \in \text { region } 1 \\
0 & \rho \in \text { region } 2
\end{array}\right.
$$


TAP - manuscript: versatile 2D schemes composite objects by Ivan Sekulic et al. UPC

$$
\boldsymbol{C}_{2}(\boldsymbol{\rho})=\left\{\begin{array}{cc}
0 & \boldsymbol{\rho} \in \text { region } 1 \\
\boldsymbol{C}_{2}^{S}(\boldsymbol{\rho}) & \boldsymbol{\rho} \in \text { region } 2
\end{array}\right.
$$

The electric and magnetic currents, $\boldsymbol{J}_{i}$ and $\boldsymbol{M}_{i}$ are expanded with tangentially orientated $\left\{\boldsymbol{f}_{1}^{1} \ldots \boldsymbol{f}_{N s}^{1}, \boldsymbol{f}_{1}^{2} \ldots \boldsymbol{f}_{N s}^{2}\right\}(2)$, and z-oriented $\left\{\boldsymbol{g}_{1}^{1} \ldots \boldsymbol{g}_{N s}^{1}, \boldsymbol{g}_{1}^{2} \ldots \boldsymbol{g}_{N s}^{2}\right\}$ discontinuous piecewise linear sets of basis functions, such that

$$
\begin{aligned}
\boldsymbol{M}_{i} & \simeq \sum_{p=1}^{2} \sum_{n=1}^{N s} M_{n}^{p, i} \boldsymbol{g}_{n}^{p} \quad i=1,2 \\
\boldsymbol{J}_{i} & \simeq \sum_{p=1}^{2} \sum_{n=1}^{N s} J_{n}^{p, i} \boldsymbol{f}_{n}^{p} \quad i=1,2
\end{aligned}
$$

where the sequences $\left\{J_{n}^{1, i}\right\},\left\{J_{n}^{2, i}\right\}$ and $\left\{M_{n}^{1, i}\right\},\left\{M_{n}^{2, i}\right\}$ denote the unknown coefficients in the expansion of the electric and magnetic current, respectively, and

$$
\boldsymbol{g}_{n}^{p}\left(\boldsymbol{\rho}^{\prime}\right)=\frac{1}{h_{n}}\left[\left(\boldsymbol{\rho}^{\prime}-\boldsymbol{\rho}_{n}^{p}\right) \cdot \hat{l}_{n}\right] \hat{z} \quad \boldsymbol{\rho}^{\prime} \in L_{n} \quad p=1,2
$$
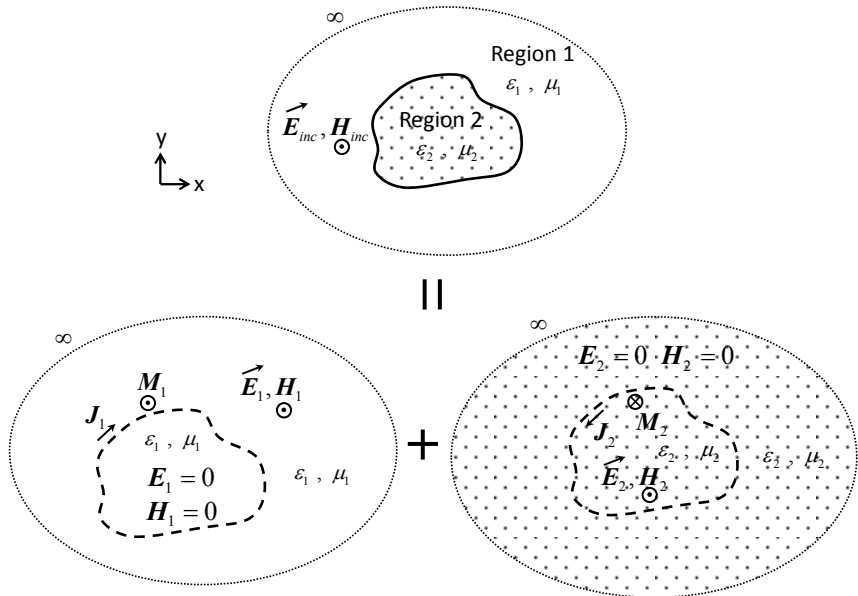

Fig. 3. Application of the surface equivalence theorem to a $z$-infinite penetrable cylinder with arbitrary section under an impinging TE-polarized plane wave.

The discretization of TE-PMCHWT relies on the approximated scattered electric and magnetic fields in the problems associated with region $i, \tilde{\boldsymbol{E}}_{i}^{S}, \tilde{\boldsymbol{H}}_{i}^{S}$ which yield

$$
\begin{gathered}
\tilde{\boldsymbol{E}}_{i}^{S}=-\sum_{p=1}^{2} \sum_{n=1}^{N s} j k_{i} \eta_{i} \boldsymbol{A}_{n}^{p}\left(k_{i}, \boldsymbol{\rho}\right) J_{n}^{p, i}-\sum_{p=1}^{2} \sum_{n=1}^{N s} \nabla \Phi_{n}^{p}\left(k_{i}, \boldsymbol{\rho}\right) J_{n}^{p, i} \\
-\sum_{p=1}^{2} \sum_{n=1}^{N s} \boldsymbol{R}_{n}^{p}\left(k_{i}, \boldsymbol{\rho}\right) \times \hat{z} M_{n}^{p, i} \quad i=1,2 \\
\tilde{\boldsymbol{H}}_{i}^{S}=\sum_{p=1}^{2} \sum_{n=1}^{N s} \boldsymbol{R}_{n}^{p}\left(k_{i}, \boldsymbol{\rho}\right) \times \hat{l}_{n} J_{n}^{p, i}-\sum_{p=1}^{2} \sum_{n=1}^{N s} j \frac{k_{i}}{\eta_{i}} \boldsymbol{F}_{n}^{p}\left(k_{i}, \boldsymbol{\rho}\right) M_{n}^{p, i} \\
i=1,2
\end{gathered}
$$

The quantities $\eta_{i}$ and $k_{i}$ represent, respectively, the wave impedance and the wave number of the $i$-th region and the potentials $\boldsymbol{F}_{n}^{p}$ and $\boldsymbol{R}_{n}^{p}$ are defined as

$$
\begin{gathered}
\boldsymbol{F}_{n}^{p}\left(k_{i}, \boldsymbol{\rho}\right)=\int_{L_{n}} G_{k_{i}}\left(\boldsymbol{\rho}, \boldsymbol{\rho}^{\prime}\right) \boldsymbol{g}_{n}^{p}\left(\boldsymbol{\rho}^{\prime}\right) d l^{\prime} \\
\boldsymbol{R}_{n}^{p}\left(k_{i}, \boldsymbol{\rho}\right)=\int_{L_{n}} \frac{1}{h_{n}}\left[\left(\boldsymbol{\rho}^{\prime}-\boldsymbol{\rho}_{n}^{p}\right) \cdot \hat{l}_{n}\right] \nabla G_{k_{i}}\left(\boldsymbol{\rho}, \boldsymbol{\rho}^{\prime}\right) d l^{\prime} \\
p=1,2 \quad i=1,2
\end{gathered}
$$

where $G_{k_{i}}$ denotes the Green's function corresponding to the homogeneous problem associated with the $i$-th region.

The electric field in (22) exhibits hypersingular Kernel contributions over the boundary line, associated with the terms $\nabla \Phi_{n}^{p}\left(k_{i}, \rho\right)$. In contrast, the magnetic field in (23) does not show such contributions because of the inherent zero magnetic charge density in the TE-scattering problems. In consequence, whereas the scattered magnetic fields can be Galerkin-tested over the boundary line, the testing of the scattered electric fields needs to be shifted off the boundary line, inside the region where, in light of the equivalence principle, the electric and magnetic fields must be null (see Fig. 3). We assume different values of relative permittivity $\left(\varepsilon_{r, 1} \neq \varepsilon_{r, 2}\right)$ and same relative permeability $\left(\mu_{r, 1}=\mu_{r, 2}=1\right)$ in both regions, whereby $k_{i}=k_{0} \sqrt{\varepsilon_{r, i}} \quad$ and $\eta_{i}=\eta_{0} / \sqrt{\varepsilon_{r, i}} \quad(i=1,2)$, where $k_{0}, \eta_{0}$ denote, respectively, the free-space wavenumber and impedance.

The Galerkin-testing of the magnetic-field boundary equation at the boundary line results in

$$
\begin{array}{r}
\int_{L_{m}} \boldsymbol{g}_{m}^{q} \cdot \boldsymbol{H}_{i n c} d l=\int_{L_{m}} \boldsymbol{g}_{m}^{q} \cdot\left(\tilde{\boldsymbol{H}}_{2}^{S}-\tilde{\boldsymbol{H}}_{1}^{S}\right) d l \\
q=1,2 \quad 1 \leq m \leq N s
\end{array}
$$

where the limiting values of the singular Kernel contributions in (25), at both sides of the boundary line, are cancelled out so that such integrals can be taken as Cauchy principal values.

In this paper, we present two nonconforming MoM-discretizations of the TE-PMCHWT. In both cases we apply the magnetic-field boundary equation in (26). As regards the electric-field boundary equation, we apply in one case the surface testing scheme, which we call TE-PMCHWT[surf], and in the other case the tangential-normal testing, TE-PMCHWT[tn]. In view of (9) and (10), the tested electric-field boundary equation in TE-PMCHWT[surf] yields

$$
\begin{gathered}
\iint_{S_{m}^{2}} \boldsymbol{P}_{m}^{2, q} \cdot \boldsymbol{E}_{i n c} d S=\iint_{S_{m}^{1}} \boldsymbol{P}_{m}^{1, q} \cdot \tilde{\boldsymbol{E}}_{2}^{S} d S-\iint_{S_{m}^{2}} \boldsymbol{P}_{m}^{2, q} \cdot \tilde{\boldsymbol{E}}_{1}^{S} d S \\
q=1,2 \quad 1 \leq m \leq N S
\end{gathered}
$$

where the set of testing functions $\left\{\boldsymbol{P}_{1}^{\mathbf{1 , 1}} \ldots \boldsymbol{P}_{N s}^{\mathbf{1 , 1}}, \boldsymbol{P}_{1}^{\mathbf{1 , 2}} \ldots \boldsymbol{P}_{N s}^{\mathbf{1 , 2}}\right\}$ and $\left\{\boldsymbol{P}_{1}^{\mathbf{2}, 1} \ldots \boldsymbol{P}_{N s}^{\mathbf{2}, 1}, \boldsymbol{P}_{1}^{\mathbf{2}, 2} \ldots \boldsymbol{P}_{N s}^{\mathbf{2}, 2}\right\}$ adopt the definition in (7) over a set of quadrilaterals, respectively, $\left\{S_{m}^{1}\right\}$ and $\left\{S_{m}^{2}\right\}$, attached to the boundary segmentation, inside regions 1 and 2 (see Fig. 4). 
Similarly, in light of (14) and (15), the electric-field boundary equation in TE-PMCHWT[tn] becomes

$$
\begin{gathered}
\int_{L_{m} \cup N_{m}^{2, q+1}} \boldsymbol{t}_{m}^{2, q} \cdot \boldsymbol{E}_{i n c} d l=\int_{L_{m} \cup N_{m}^{1, q+1}} \boldsymbol{t}_{m}^{1, q} \cdot \tilde{\boldsymbol{E}}_{2}^{S} d l-\int_{L_{m} \cup N_{m}^{2, q+1}} \boldsymbol{t}_{m}^{2, q} \cdot \tilde{\boldsymbol{E}}_{1}^{S} d l \\
q=1,2 \quad 1 \leq m \leq N S
\end{gathered}
$$

where the set of testing functions $\left\{\boldsymbol{t}_{1}^{1,1} \ldots \boldsymbol{t}_{N s}^{1,1}, \boldsymbol{t}_{1}^{1,2} \ldots \boldsymbol{t}_{N s}^{1,2}\right\}$ and $\left\{\boldsymbol{t}_{1}^{2,1} \ldots \boldsymbol{t}_{N s}^{2,1}, \boldsymbol{t}_{1}^{2,2} \ldots \boldsymbol{t}_{N s}^{2,2}\right\}$ adopt the definition in (12) in such a manner that the sets of segments $\left\{N_{1}^{1,1} \ldots N_{N s}^{1,1}, N_{1}^{1,2} \ldots N_{N s}^{1,2}\right\}$ and $\left\{N_{1}^{2,1} \ldots N_{N s}^{2,1}, N_{1}^{2,2} \ldots N_{N s}^{2,2}\right\}$ are oriented from the endpoints of the boundary segments $\left\{L_{1} \ldots L_{N s}\right\}$ into, respectively, regions 1 and 2 (see Fig. 4). Note that in both testing schemes, (27) and (28), the incident and scattered fields are tested in the regions where, in accordance with the equivalence theorem, the total fields must be zero. The performance of such implementations depends on the heights of the testing domains $(H)$, which we set in terms of the length of the associated segments $(h)$ (see Figs. 1, 2 and 4). In this paper, we impose the same value of $H$ for each of the testing domains, in regions 1 or 2, attached to a particular boundary segment.

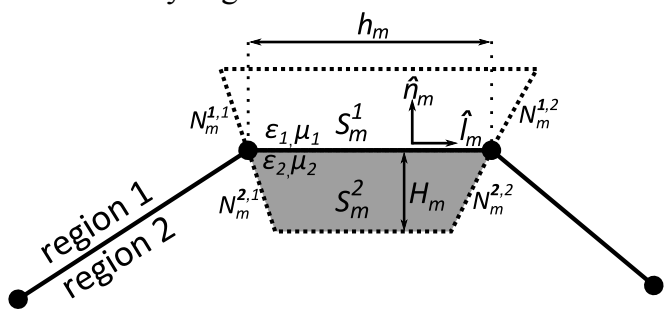

Fig. 4. $m$ th segment arising from the discretized section of an infinitely long penetrable cylinder where the testing schemes are defined conformal to the boundary.

Our nonconforming implementation of the TM-PMCHWT can be readily obtained from the expressions above. Indeed, in the TM case, the electromagnetic wave is impinging on the object so that the magnetic field is oriented transversally and the electric field is z-oriented. In consequence, the sets of basis functions adopted for our nonconforming TM-expansions of the electric and magnetic currents arise from the sets of functions employed in the TE-expansions of, respectively, the magnetic and electric currents. Similarly, since in the TM case the electric charges are zero, the electric-field boundary equation is Galerkin-tested, whereas the surface or the tangential-normal testing needs to be applied to the magnetic-field boundary equation, giving thus rise to the implementations TM-PMCHWT[surf] or TM-PMCHWT[tn].

\section{V.NONCONFORMING EFIE-PMCHWT FOR COMPOSITE PEC AND PENETRABLE STRUCTURES WITH JUNCTIONS}

Our nonconforming implementations for the TE-scattering analysis of composite infinitely long objects, with perfectly conducting or penetrable regions, TE-EFIE-PMCHWT, arise from: (a) the definition of the expansions in (20) for the electric currents and the imposition of the electric-field boundary conditions (27) or (28) over all the boundary interfaces ; (b) the definition of the expansion (19) for the magnetic currents and the imposition of the Galerkin-tested magnetic-field boundary equation (26), exclusively, over interfaces shared by penetrable regions (see Fig. 5). The electric-field boundary conditions (27) and (28) when applied to boundary lines bordering on perfectly conducting regions need to consider only the scattered-field or incident-field contributions associated with the penetrable region.

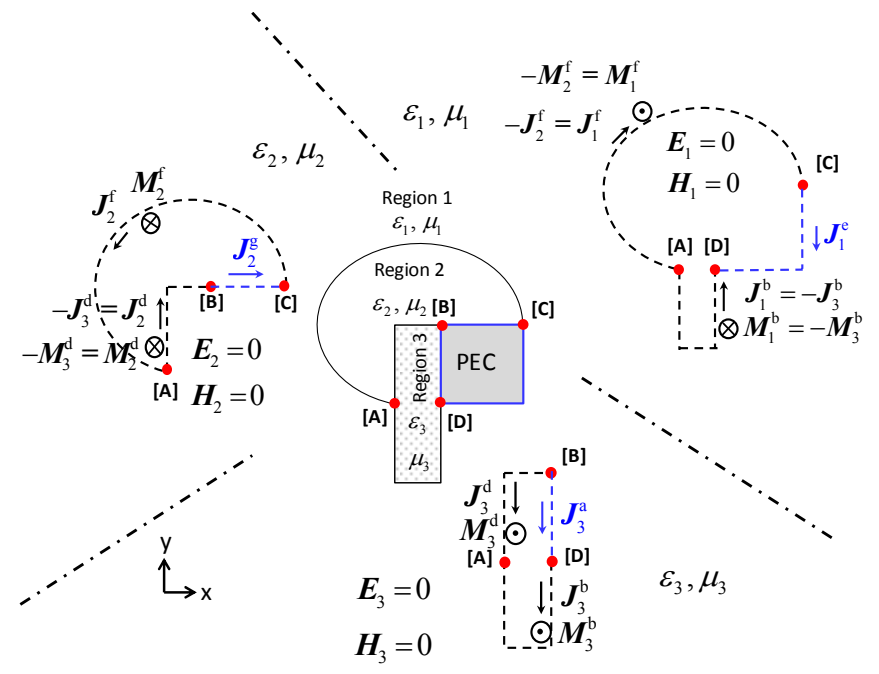

Fig. 5. Surface equivalence theorem applied to a composite object with two penetrable regions and one PEC region. The black and blue dashed lines denote, respectively, line interfaces between penetrable regions or on PEC regions. The red nodes denote junctions.

In general, the continuous piecewise linear implementation of the TE-EFIE-PMCHWT formulation is defined in an analogous manner as the nonconforming implementation for basis and testing functions out of junctions, except for the fact that the electric and magnetic field conditions are Galerkin tested. This scheme treats the interface between two different regions as single lines but the continuous piecewise linear implementations become somewhat laborious at junction nodes, where specially tailored basis and testing functions need to be defined, namely, oriented $R W G$ [18] or multiplet [19]. In Fig. 5, we show a composite structure with one penetrable junction $[\mathrm{A}]$, where all the intersecting regions are penetrable, and three PEC-penetrable junctions [B], [C] and [D], where one of the intersecting regions is PEC. In both types of junctions, electric field and current continuity conditions around junctions need to be enforced; moreover, for the penetrable junction the magnetic continuity of the current and field conditions are also required. Note that, unlike the single-object penetrable case, where each unknown (electric or magnetic) is invoked by the two homogeneous penetrable problems, the unknowns associated with the penetrable junction-node in Fig. 5 are invoked by the three associated homogeneous problems, one 
for each intersecting region at the junction. Similarly, the (electric) unknowns at each PEC-penetrable junction-node [B], [C] or [D] are invoked by different pairs of associated homogeneous problems, respectively, regions $2,31,2$ or 1,3. Clearly, the node-based single-line TE-EFIE-PMCHWT implementation involves a significant burden when handling junctions if compared with our segment-based discretization, which by definition ignores junctions.

Interestingly, another conforming implementation of TE-EFIE-PMCHWT can be obtained by analyzing the composite structure as a set of disjoint objects, corresponding to each of the regions of the composite object, immersed in the free-space and with separation distances tending to zero $(\delta \rightarrow 0)$ (see Fig. 6) [20]. This scheme considers the interfaces as $t w o$ contact lines and becomes easier to implement than the single-line approach since no continuity exceptions need to be considered at junction nodes. However, the amount of unknowns is increased because redundant currents are defined over the two contact surfaces. Also, in addition to the Cauchy principal value of the integrals, the residue term of (25) needs to be computed and for some particular examples the multi-body modelling may become complicated.

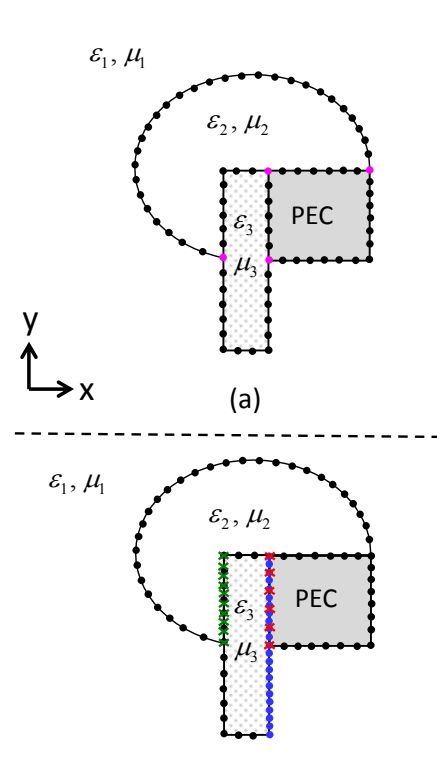

(c)

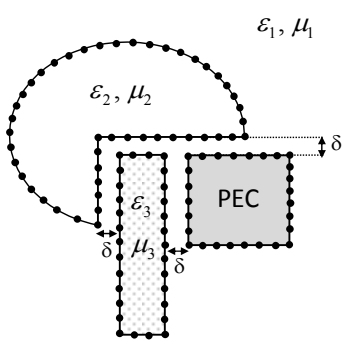

(b)

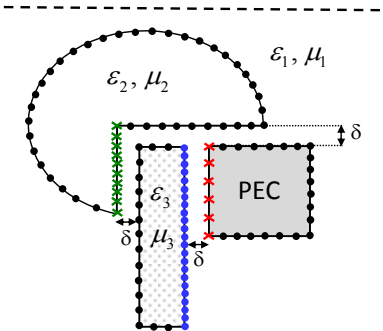

(d)
Fig. 6. Analysis of composite objects: (a) single-line interfaces and conformal segmentations; (b) interfaces with two contact lines and conformal segmentations when $\delta \rightarrow 0$; (c) single-line interfaces and nonconformal segmentations; (d) interfaces with two contact lines and nonconformal segmentations when $\delta \rightarrow 0$.

\section{NUMERICAL RESULTS}

In subsection A, we show TE-scattering results, RCS and near field, for infinitely long homogeneous dielectric objects with sharp-edged sections immersed in the free space, with small or moderate electrical dimensions, and several dielectric contrasts. We show the improved accuracy versus the number of unknowns $(N)$ and the height of the testing domains $(H)$ of the corresponding nonconforming implementations, TE-PMCHWT[surf] and TE-PMCHWT[tn], with respect to the conventional continuous implementation, TE-PMCHWT[C]. Accuracy plots for our nonconforming TM-PMCHWT implementations and single dielectrics are omitted because no singular-field performance occurs at edges [34].

In subsection B, we show RCS results for infinitely long composite objects inside the free space, with piecewise homogeneous regions, PEC or dielectric. We show how our nonconforming implementations of TE-EFIE-PMCHWT or TM-EFIE-PMCHWT for composite bodies, where the nodes are ignored in both the current expansion and field testing, allow an agile management of junction nodes while providing similar or improved accuracy. Furthermore, these implementations allow an easy management of composite objects arising from the juxtaposition of penetrable targets meshed with independent segmentations, which are typically nonconformal (i.e. with nonmatching nodes) (see Fig. 6.c). Although in theory the two-contact-line approach can handle such problems (see Fig. 6.d), in practice the required computation of the residue terms becomes bothersome when the field- and source- segmentations do not match. For such composite multi-penetrable structures, our nonconforming TE-PMCHWT or TM-PMCHWT implementations allow the definition of the electric current and the electric-field boundary condition at one nonmatching mesh and the magnetic current and the magnetic-field boundary condition over the other nonmatching mesh. This is still a single-line approach because the field equations and the currents are defined at one boundary line, but is meshed through two different, overlapped, segmentations.

In all the test cases, we compute the inner line integrals in the generation of the impedance matrix through the singularity subtraction technique and 6-point quadrature rule. In EFIE-PMCHWT[surf], we compute the surface testing over each quadrilateral through surface integrals over two triangles with 3-point Gaussian evaluations. In EFIE-PMCHWT[C] and EFIE-PMCHWT[tn] the outer line integrals are computed with, respectively, 2 and 4-point rules, for the potentials $\boldsymbol{A}, \boldsymbol{F}$ or $\Phi$ and with, respectively, 4 and 8-point rules for $\boldsymbol{R}$. In all the testing objects, the scattered fields are computed under an impinging $+y$ propagating plane wave and the free-space wavelength $\lambda_{0}$ is set to $0.06 \pi \mathrm{m}$.

\section{A. Single Objects}

We assess the relative performance of the TE-PMCHWT implementations by displaying the root-mean-square ( $\mathrm{rms}$ ) relative error of the near-field and RCS results. All the testing domains are defined conformal to the boundary. We define the near-field relative error, $e_{\text {near }}$, for a particular implementation by computing the approximated electric and magnetic fields, respectively, $\tilde{\boldsymbol{E}}$ and $\tilde{\boldsymbol{H}}$, over a set of $M$ points $\left\{\boldsymbol{\rho}_{1}, \ldots, \boldsymbol{\rho}_{M}\right\}$ such that

$$
e_{\text {near }}=\frac{\left[\sum_{j=1}^{M}\left|\tilde{\boldsymbol{E}}\left(\boldsymbol{\rho}_{j}\right)-\boldsymbol{E}_{R E F}\left(\boldsymbol{\rho}_{j}\right)\right|^{2}+\eta_{0}^{2} \sum_{j=1}^{M}\left|\tilde{\boldsymbol{H}}\left(\boldsymbol{\rho}_{j}\right)-\boldsymbol{H}_{R E F}\left(\boldsymbol{\rho}_{j}\right)\right|^{2}\right]^{1 / 2}}{\left[\sum_{j=1}^{M}\left|\boldsymbol{E}_{R E F}\left(\boldsymbol{\rho}_{j}\right)\right|^{2}+\eta_{0}^{2} \sum_{j=1}^{M}\left|\boldsymbol{H}_{R E F}\left(\boldsymbol{\rho}_{j}\right)\right|^{2}\right]^{1 / 2}}
$$


where the observation points are distributed uniformly around the object section at a very close distance (the mesh parameter adopted in our nonconforming implementations). Similarly, we define the far-field error, $e_{\text {far }}$, in terms of the bistatic RCS computed with a particular implementation over a set of $M$ observation angles $\left\{\theta_{1}, \ldots, \theta_{M}\right\}$, uniformly distributed, so that

$$
e_{f a r}=\frac{\left[\sum_{j=1}^{M}\left|R C S\left(\theta_{j}\right)-R C S_{R E F}\left(\theta_{j}\right)\right|^{2}\right]^{1 / 2}}{\left[\sum_{j=1}^{M}\left|R C S_{R E F}\left(\theta_{j}\right)\right|^{2}\right]^{1 / 2}}
$$

where $\theta_{1}=0$ and $\theta_{M}=2 \pi-2 \pi / M$. The near- and far-field errors provide relative measures with respect to reference near-field and RCS solutions, respectively, $\boldsymbol{E}_{R E F}, \boldsymbol{H}_{R E F}$, and $R C S_{R E F}$. With no analytical solutions available for the sharp-edged objects tested, the reference solutions in (29) and (30) are computed with the conventional implementation, TE-PMCHWT[C], and very fine degree of meshing of the boundary line (around 15000 or 20000 segments for the tested objects with, respectively, small or moderate electrical dimensions). The segmentations of the boundary line of the tested objects when computing the reference results employ geometrical mesh refinement near the sharp edges so that the ratio of the smallest segment to the biggest segment is set to 0.05 . The source integrals in the computed far and near fields in (29) and (30) are carried out with 2-point and 10-point numerical rules, respectively. In all the examples, we adopt $M=1000$.

1) Accuracy versus $H$ : In Figs. 7-10 we plot the error performance of our nonconforming TE-PMCHWT implementations for two sharp-edged dielectric objects with several dielectric contrasts (with relative permittivities of 5,15 , $50,90)$ in terms of the height of the testing domains, $H$, defined as a fraction of the mesh parameter $h$. We analyze two infinitely long objects with equilateral triangular sections and sides of $0.2 \lambda_{0}$ (Figs. 7 and 8 ) and $1.9 \lambda_{0}$ (Figs. 9 and 10). We display the normalized error $\bar{e}$ for each nonconforming implementation of TE-PMCHWT. The far-field normalized error, $\bar{e}_{f a r}$, is shown in Figs. 7 and 9, whereas we plot the near-field normalized error, $\bar{e}_{\text {near }}$, in Figs. 8 and 10. We define these normalized errors as

$$
\begin{gathered}
\bar{e}_{\text {near }}^{\text {tn }}=e_{\text {near }}^{\text {th }} / e_{\text {near }}^{C}, \quad \bar{e}_{\text {near }}^{\text {surf }}=e_{\text {near }}^{\text {sur }} / e_{\text {near }}^{C} \\
\bar{e}_{\text {far }}^{\text {th }}=e_{\text {far }}^{t n} / e_{\text {far }}^{C}, \quad \bar{e}_{\text {far }}^{\text {surf }}=e_{\text {far }}^{\text {surf }} / e_{\text {far }}^{C}
\end{gathered}
$$

where $e_{\text {near }}^{\text {tn }}, e_{\text {near }}^{\text {surf }}$ and $e_{\text {far }}^{t n}, e_{\text {far }}^{\text {surf }}$ arise from computing, respectively, (29) and (30), with the corresponding nonconforming implementations of TE-PMCHWT. Similarly, $e_{\text {near }}^{C}$ and $e_{\text {far }}^{C}$ are derived from the evaluation of (29) and (30), respectively, with the solution of TE-PMCHWT[C]. In order to build a fair comparison, our nonconforming and the piecewise continuous implementations handle the same number of unknowns. Hence, TE-PMCHWT[C] makes use of segments that are twice smaller than the segments adopted in our nonconforming implementations, where two unknowns are assigned to each boundary segment. Also, we establish uniform segmentations of the boundary line so that the mesh parameters are similar in all cases $\left(h \approx 0.0033 \lambda_{0}\right.$ for TE-PMCHWT[C]).

In view of Figs. 7-10, our nonconforming implementations TE-PMCHWT[surf] or TE-PMCHWT[tn] outperform TE-PMCHWT[C] for a wide range of $H$ values in both far and near-field scenarios. In view of the definition of the normalized errors in (31) and (32), there is improvement of our nonconforming implementations as long as the normalized error is smaller than 1. As predicted by the theory [34], the well-performing $H$ ranges in all the tests grow as the relative dielectric contrast rises. From the comparison of far-field errors in Figs. 7 and 9 with the near-field errors in Figs. 8 and 10, the $H$-ranges of improved far-field appear similar or slightly wider than the near-field $H$-ranges. Particularly, for the case of highest contrast, whereas the far-field tests show improved performance for $H$ values between $h / 20$ and $h / 1500$, for the small section, or between $h / 20$ and $h / 660$ for the moderate section, the improved $H$-performance in the near-field tests lies, respectively, between $h / 10$ and $h / 250$ or between $h / 6$ and $h / 260$. Furthermore, we see that the impact on the overall accuracy of our nonconforming implementations of TE-PMCHWT due to the surface testing and the tangential-normal testing is quite similar. Interestingly, our nonconforming discretization of the magnetic current in (19) and the Galerkin-testing of the magnetic-field equation in (26) can be also implemented with a set of piecewise uniform basis functions. Our tests show that the general trend with regard to the observed improved performance for growing dielectric contrasts prevails.

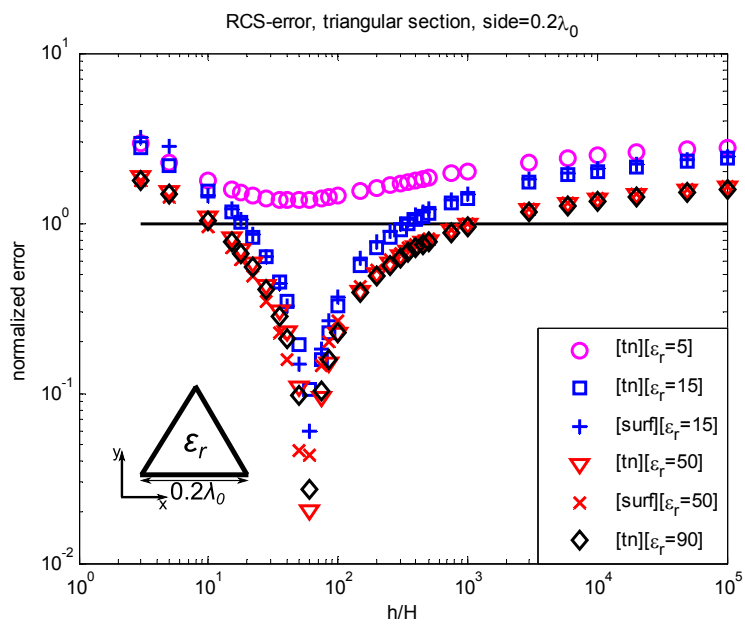

Fig. 7. Normalized RCS error (32) computed for several nonconforming PMCHWT implementations, relative to PMCHWT[C], and same number of unknowns (360) versus the height $H$ of the testing domains for a dielectric cylinder with equilateral triangular section, side $0.2 \lambda_{0}\left(\lambda_{0}=0.06 \pi \mathrm{m}\right)$ and several relative permittivities $(5,15,50,90)$. The reference results are computed with TE-PMCHWT[C] and very fine meshing (12000 segments). 


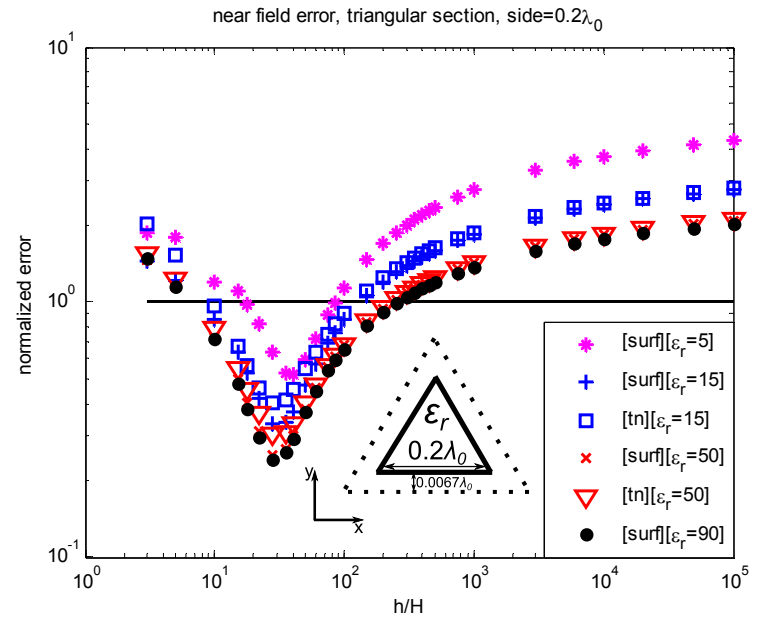

Fig. 8. Normalized near-field error (31) computed for several nonconforming PMCHWT implementations, relative to PMCHWT[C], and same number of unknowns (360) versus the height $H$ of the testing domains for a dielectric cylinder with equilateral triangular section, side $0.2 \lambda_{0}\left(\lambda_{0}=0.06 \pi \mathrm{m}\right)$ and several relative permittivities $(5,15,50,90)$. The reference results are computed with TE-PMCHWT[C] and very fine meshing (12000 segments).

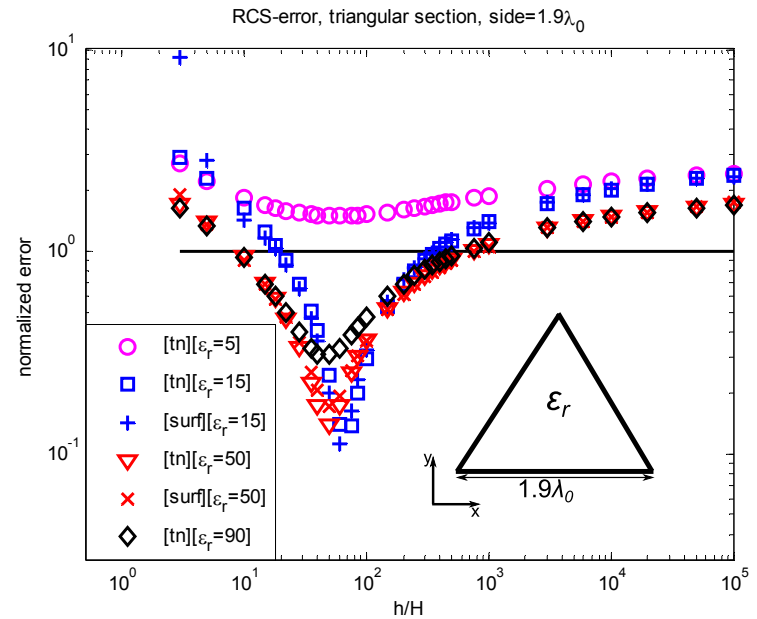

Fig. 9. Normalized RCS error (32) computed for several nonconforming PMCHWT implementations, relative to PMCHWT[C], and same number of unknowns (3480) versus the height $H$ of the testing domains for a dielectric cylinder with equilateral triangular section, side $1.9 \lambda_{0}\left(\lambda_{0}=0.06 \pi \mathrm{m}\right)$ and several relative permittivities $(5,15,50,90)$. The reference results are computed with TE-PMCHWT[C] and very fine meshing (18000 segments).

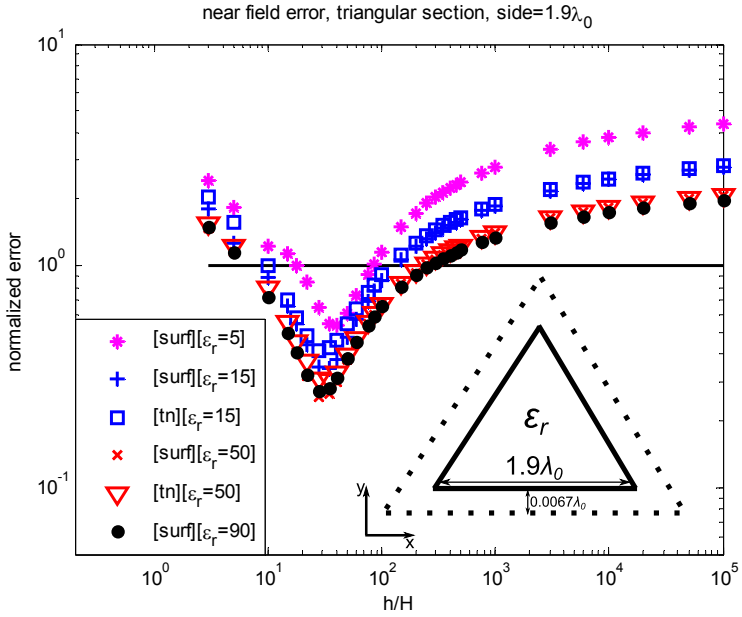

Fig. 10. Normalized near-field error (31) computed for several nonconforming PMCHWT implementations, relative to PMCHWT[C], and same number of unknowns (3480) versus the height $H$ of the testing domains for a dielectric cylinder with equilateral triangular section, side $1.9 \lambda_{0}\left(\lambda_{0}=0.06 \pi \mathrm{m}\right)$ and several relative permittivities $(5,15,50,90)$. The reference results are computed with TE-PMCHWT[C] and very fine meshing (18000 segments).

2) Accuracy versus $N$ : We show relative far- and near-field errors, respectively, in Figs. 11, 13 and Figs. 12, 14, as defined in (30) and (29), for TE-PMCHWT[surf], TE-PMCHWT[tn] and TE-PMCHWT[C] in terms of the number of unknowns $(N)$ for infinitely long dielectric cylinders and a relative permittivity of 50 with square or equilateral triangular sections and sides of $0.2 \lambda_{0}$ and $1.9 \lambda_{0}$. Our nonconforming implementations of TE-PMCHWT employ $H$ values that are inside the good performing $H$-ranges. As regards the far-field behavior, in all cases, in view of Figs. 11 and 13, TE-PMCHWT[C] exhibits around $\mathrm{O}\left(h^{1.3}\right)$ decrease in the far-field error as the mesh parameter $h$ is reduced. As shown in Fig. 11 for the electrically small cylinders, our nonconforming implementations exhibit improved convergence trends in the far-field error reduction, around $\mathrm{O}\left(h^{2}\right)$ for the square section and $\mathrm{O}\left(h^{1.7}\right)$ for the triangular section. Similarly, our nonconforming implementations of TE-PMCHWT for the electrically moderate cylinders in Fig. 13 exhibit decrease in the far-field error around $\mathrm{O}\left(h^{2.6}\right)$ for the square section and $\mathrm{O}\left(h^{2.8}\right)$ for the triangular section. As for the near-field performance, in all cases, in view of Figs. 12 and 14, TE-PMCHWT[C] exhibits around $\mathrm{O}\left(h^{1.5}\right)$ decrease in the near-field error. Our nonconforming implementations of TE-PMCHWT, when compared with TE-PMCHWT[C], provide faster reduction in the near-field error decrease as $h$ diminishes. Indeed, whereas the electrically small cylinders in Fig. 12 exhibit around $\mathrm{O}\left(h^{2}\right)$ error reduction, the electrically moderate cylinders in Fig. 14 show a steeper reduction, around $\mathrm{O}\left(h^{2.4}\right)$. 


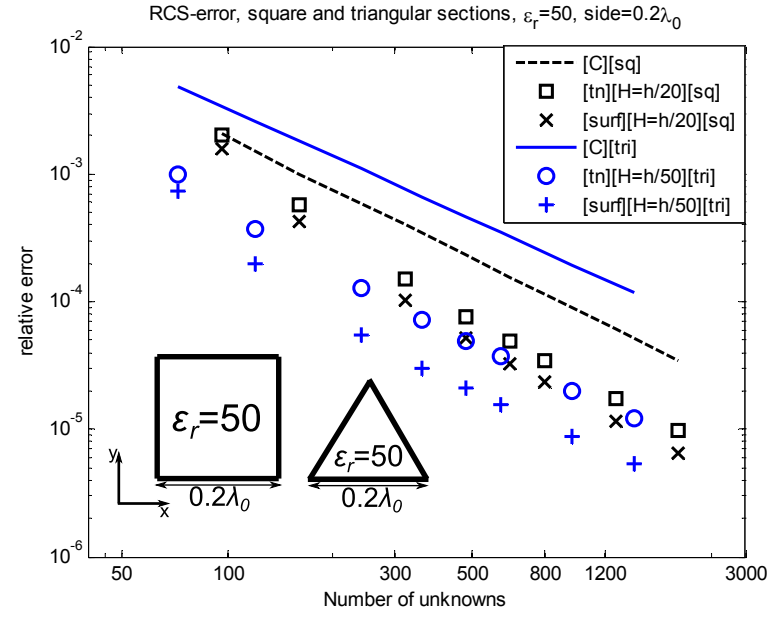

Fig. 11. RCS relative error computed for several nonconforming PMCHWT implementations versus the number of unknowns for dielectric cylinders with square and equilateral triangular sections, side $0.2 \lambda_{0}$ and relative permittivity 50 $\left(\lambda_{0}=0.06 \pi \mathrm{m}\right)$ for several values of testing heights $H$. The reference results are computed with TE-PMCHWT[C] and very fine meshing (16000 segments for the square and 12000 for the triangle).

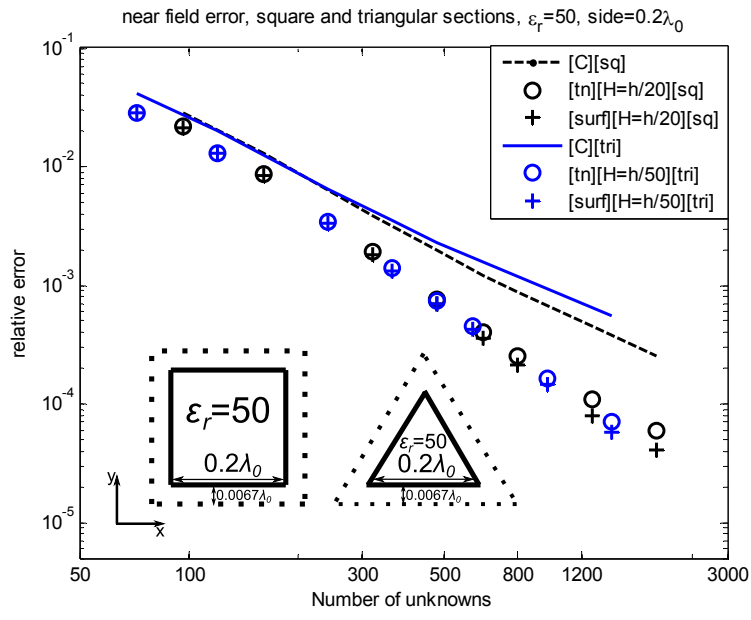

Fig. 12. Near-field relative error computed for several nonconforming PMCHWT implementations versus the number of unknowns for dielectric cylinders with square and equilateral triangular sections, side $0.2 \lambda_{0}$ and relative permittivity $50 \quad\left(\lambda_{0}=0.06 \pi \mathrm{m}\right)$ for several values of testing heights $H$. The reference results are computed with TE-PMCHWT[C] and very fine meshing (16000 segments for the square and 12000 for the triangle).

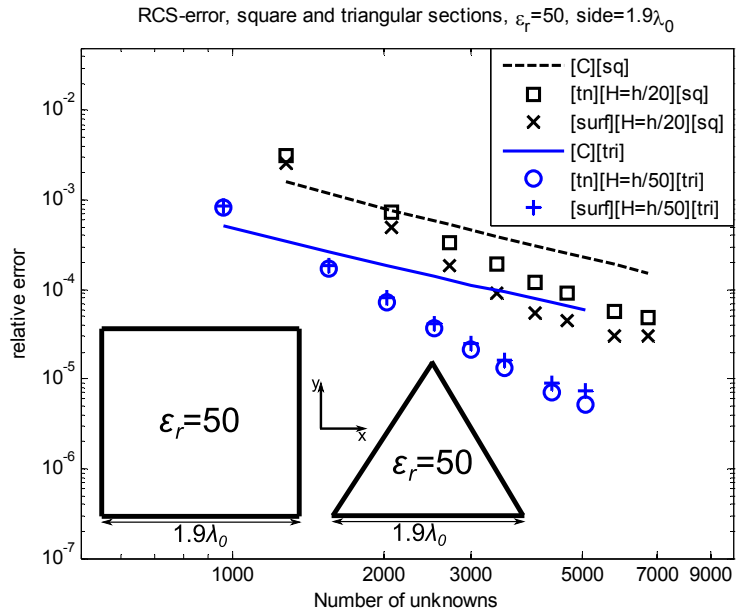

Fig. 13. RCS relative error computed for several nonconforming PMCHWT implementations versus the number of unknowns for dielectric cylinders with square and equilateral triangular sections, side $1.9 \lambda_{0}$ and relative permittivity 50 $\left(\lambda_{0}=0.06 \pi \mathrm{m}\right)$ for several values of testing heights $H$. The reference results are computed with TE-PMCHWT[C] and very fine meshing (24000 segments for the square and 18000 for the triangle).

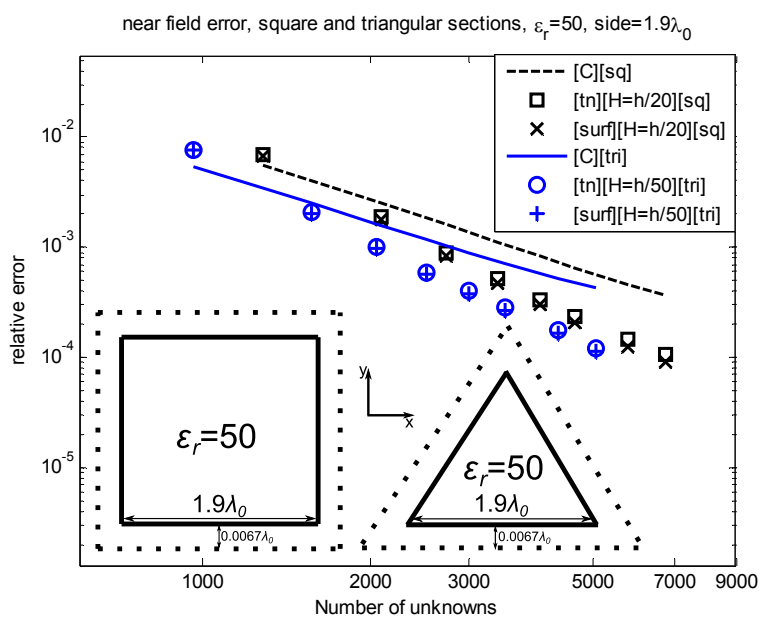

Fig. 14. Near-field relative error computed for several nonconforming PMCHWT implementations versus the number of unknowns for dielectric cylinders with square and equilateral triangular sections, side $1.9 \lambda_{0}$ and relative permittivity $50\left(\lambda_{0}=0.06 \pi \mathrm{m}\right)$ for several values of testing heights $H$. The reference results are computed with TE-PMCHWT[C] and very fine meshing (24000 segments for the square and 18000 for the triangle).

3) Hybrid PMCHWT: The hybrid implementation of TE-PMCHWT, TE-PMCHWT[hyb], in the analysis of sharp-edged infinitely long penetrable cylinders with polygonal section arises from the continuous expansion of the currents at nodes inside the sides of the polygon and the discontinuous expansion at corners [23]. This implementation is not segment-oriented and cannot hence benefit from the inherent flexibility associated with the fully discontinuous schemes. However, as shown in Fig. 15 for cylinder with equilateral triangular section and side $0.2 \lambda_{0}$ and several relative permittivities, it exhibits huge improved accuracy with respect to the fully continuous scheme (and same number of 
unknowns). Note that the hybrid and continuous PMCHWT implementations for the same boundary segmentation handle very similar amount of unknowns (the hybrid implementation accounts only for two additional unknowns at each corner node). When compared with Fig. 7 and the fully discontinuous PMCHWT implementation, it is clear that the $H$-range of improved performance for the hybrid implementation rises drastically. This illustrates that the observed trend in Fig. 7 is mainly due to the discontinuous modelling of the current transition at the corners of the sharp-nodes of the polygon, where the singular-field behavior occurs.

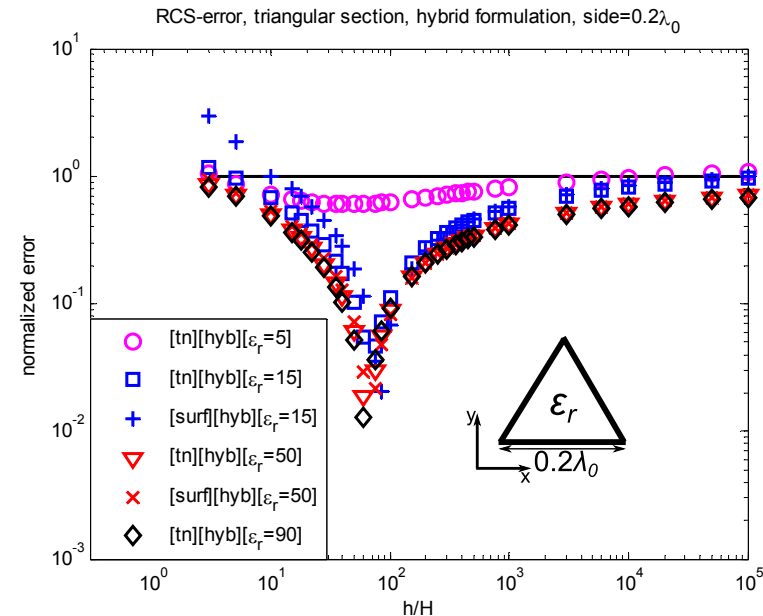

Fig. 15. Normalized RCS error (32) computed for several hybrid PMCHWT implementations, relative to PMCHWT[C], and same number of unknowns (360) versus the height $H$ of the testing domains for a dielectric cylinder with equilateral triangular section, side $0.2 \lambda_{0}\left(\lambda_{0}=0.06 \pi \mathrm{m}\right)$ and several relative permittivities $(5,15,50,90)$. The reference results are computed with TE-PMCHWT[C] and very fine meshing (12000 segments).

\section{4) Condition numbers of the impedance matrices}

In Fig. 16, we show the condition numbers of our nonconforming and conforming PMCHWT implementations versus the height $H$ of the testing domains for electrically small high-contrast dielectric cylinders and equilateral triangular or square sections. The PMCHWT implementations in Fig. 16 are scaled in order to improve the condition numbers of the resulting matrices. The magnetic-field equations are then multiplied by the free-space impedance $\eta_{0}$ and the magnetic-current density is expressed in terms of another unknown so that $\boldsymbol{M}_{i}=\eta_{0} \boldsymbol{M}_{i}^{\prime}$ [43]. In view of Fig. 16, the condition numbers resulting from surface and tangential normal testing strategies are almost the same. As the height of the testing elements $H$ diminishes, the condition numbers of our nonconforming implementations rise in comparison with the continuous piecewise linear implementation. This trend is analogous to the observed performance of the nonconforming discretization of the EFIE, with monopolar-RWG basis functions, for 3D-conductors [23].

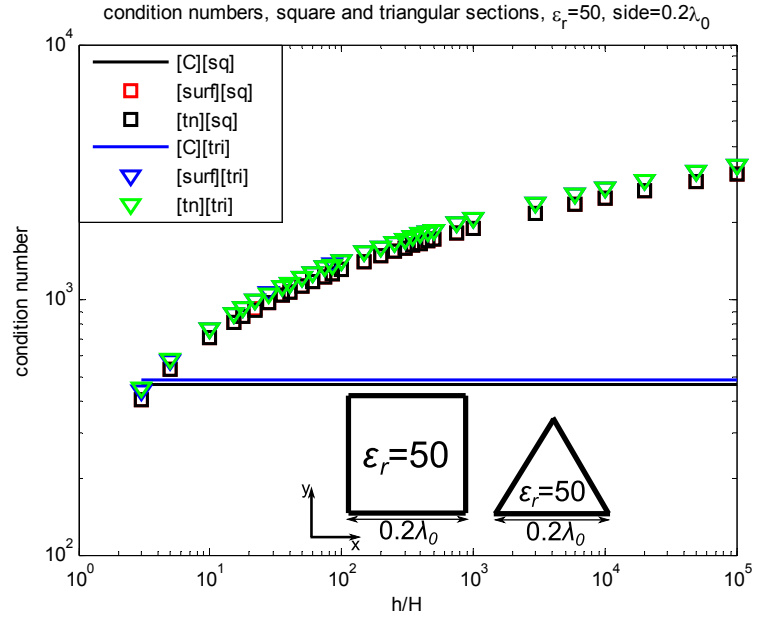

Fig. 16. Condition numbers of the impedance matrices resulting from several scaled PMCHWT implementations, discontinuous or continuous piecewise linear, with the same number of unknowns for each object versus the height $H$ of the testing elements for the dielectric cylinders with square and equilateral triangular sections, side $0.2 \lambda_{0}\left(\lambda_{0}=0.06 \pi \mathrm{m}\right)$ and relative permittivity 50 . The number of unknowns is 360 for the cylinder with triangular section and 480 for the cylinder with square section.

\section{B. Composite Objects}

For the sake of the robust and flexible analysis of complex structures, we adopt testing domains nonconformal to the boundary line whereby no a priori knowledge on nodes is required. In Fig. 17, we compare the RCS-performance of our nonconforming TE-PMCHWT implementation with testing domains defined conformal [conf] or nonconformal [nonconf] to the boundary line.

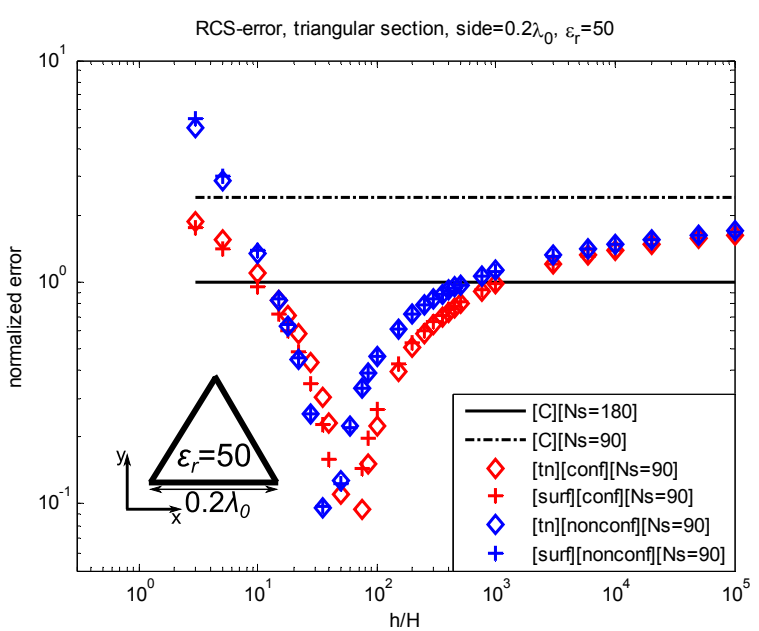

Fig. 17. Normalized RCS error (32) computed for several nonconforming PMCHWT implementations, relative to PMCHWT[C][Ns=180], versus the height $H$ of the testing domains (conformal or nonconformal to the boundary) for a dielectric cylinder with equilateral triangular section, side $0.2 \lambda_{0}$ $\left(\lambda_{0}=0.06 \pi \mathrm{m}\right)$ and $\varepsilon_{\mathrm{r}}=50$. The reference results are computed with TE-PMCHWT[C] and very fine meshing (12000 segments). Ns denotes the number of segments. 
Some loss of accuracy is observed for the nonconformal choice, which we attribute to the fact that the testing elements bordering on abruptly sharp corners cross the boundary line. We minimize such discrepancy in our nonconforming TE-EFIE-PMCHWT implementations with a testing nonconformal to the boundary by adopting very small $H$-values $(H=h / 1 e 5)$. When compared with the continuous implementation, the nonconforming implementations produce similar or better far-field results, if the same number of unknowns or the same mesh, respectively, are adopted (see Fig. 17). In Figs. 18 and 19, we show RCS results for composite objects with perfectly conducting and dielectric regions and our nonconforming implementations of TE-EFIE-PCMHWT (Figs. 18(a) and Fig. 19) or of TM-EFIE-PMCHWT implementation (Fig. 18(b)).

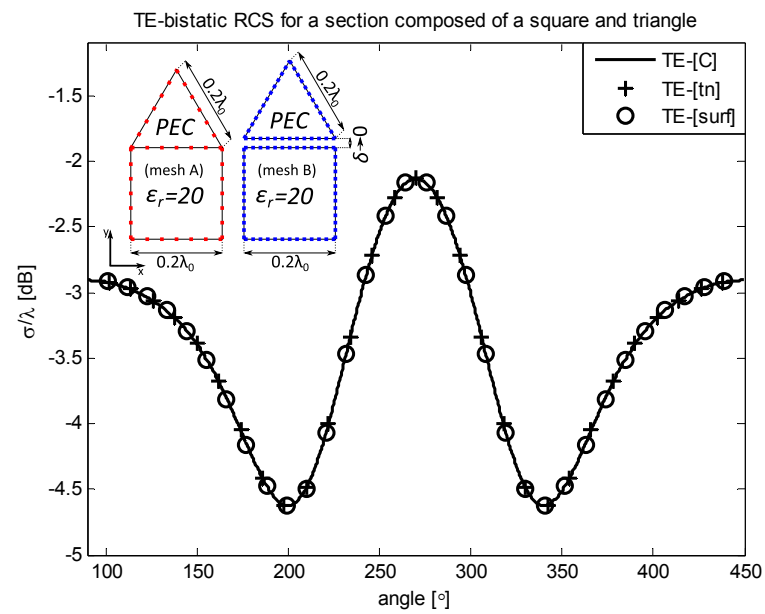

(a)

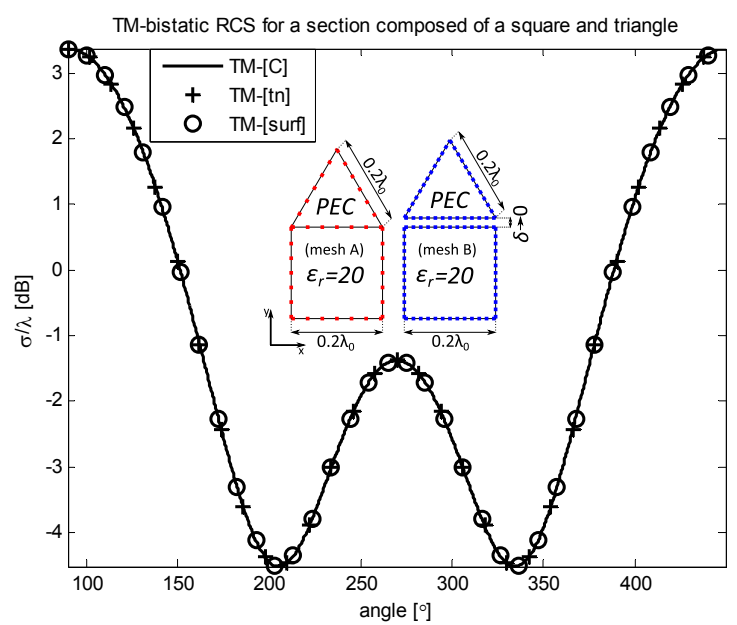

(b)

Fig. 18. Bistatic RCS of a composite object consisting of dielectric cylinder of square section and a PEC cylinder of equilateral triangle section under an impinging a) TE-polarized and b) TM-polarized +y propagating plane wave. The relative permittivity of the region with square section is 20 . The number of unknowns is 360 for our nonconforming EFIE-PMCHWT implementations and 440 for the continuous implementation, which is adopted as reference.
Whereas our EFIE-PMCHWT implementations in Figs. 18 and 19 adopt the single-line approach, the reference results are obtained with the continuous piecewise linear implementation and the two-contact-line approach. In Fig. 20, we provide TE-RCS results for a composite body with two dielectric regions arising from the juxtaposition of two independent nonconformal meshes (i.e. with nonmatching nodes). In view of Fig. 20, the single-line nonconforming TE-PMCHWT implementation provides similar accuracy as the continuous piecewise linear implementation with two contact lines. In order to reach a fair comparison, we generate the impedance matrices of our nonconforming and the piecewise linear implementations with similar sizes. In any case, the number of unknowns handled in the continuous implementations, with two contact lines, is somewhat bigger because additional unknowns are introduced where boundary lines are in contact.

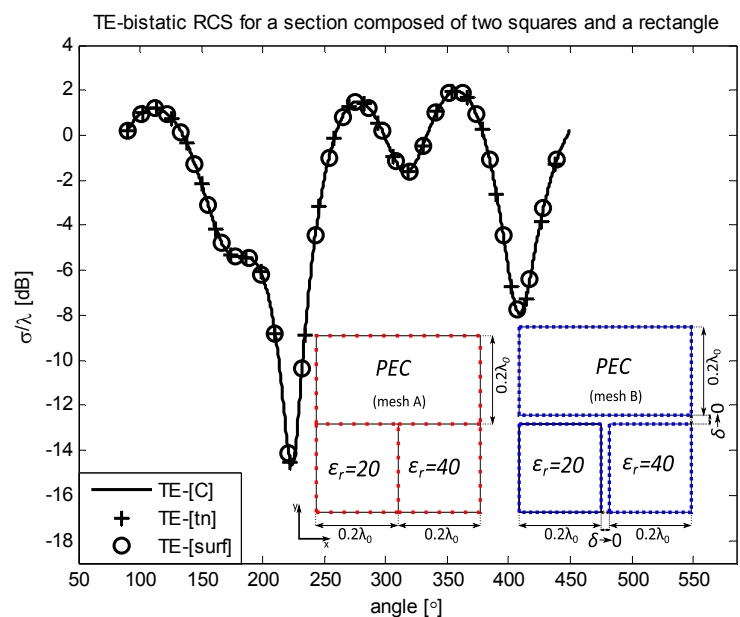

Fig. 19. Bistatic RCS of a composite object consisting of two dielectric cylinders of square section and a PEC cylinder of rectangular section under an impinging TE-polarized +y propagating plane wave. The relative permittivities of the two dielectric cylinders are 20 and 40. The number of unknowns is 640 for our nonconforming EFIE-PMCHWT implementations and 800 for the continuous implementation, which is adopted as reference.

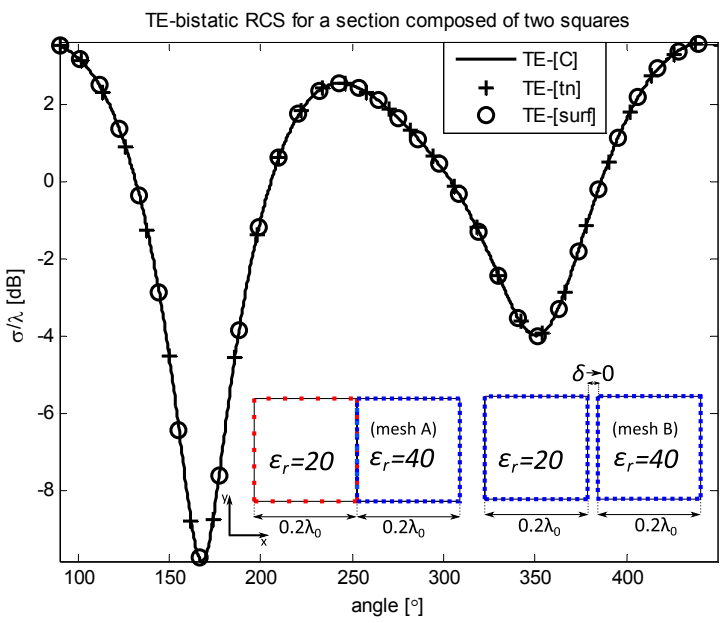

Fig. 20. Bistatic RCS of a composite object consisting of two dielectric 
cylinders of square section assembled after juxtaposing two independent closed line meshes under an impinging TE-polarized +y propagating plane wave. The relative permittivity of the two dielectric cylinders is 20 and 40 . The number of unknowns is 700 for our nonconforming PMCHWT implementations and 960 for the continuous implementation, which is adopted as reference.

\section{CONCLUSION}

The discretization with discontinuous piecewise linear basis functions of the currents and the testing of the fields off the boundary interfaces, with surface or tangential-normal testing, gives rise to new nonconforming implementations of the PMCHWT formulation for infinitely long penetrable bodies, and of the EFIE-PMCHWT formulation for infinitely long piecewise homogeneous structures, with PEC or penetrable regions. Our tests with $2 \mathrm{D}$ dielectric objects, with triangular or square sections, suggest that the nonconforming TE-analysis of sharp-edged objects with moderate or high dielectric contrasts (above 10) shows improved accuracy, versus the number of unknowns, with respect to the conventional schemes. Indeed, while the continuous piecewise linear implementation exhibits for the objects tested around $\mathrm{O}\left(h^{1.5}\right)$ decrease in RCS or near-field errors, our nonconforming implementations exhibit error decrease rates between $O\left(h^{2}\right)$ and $O\left(h^{2.5}\right)$ with an adequate choice of the height $H$ of the testing elements. Our tests show, as a general trend, that the best performing $H$-ranges lie roughly between $h / 10$ and $h / 100$. The well-performing $H$-range rises with growing values of the relative permittivity and with the reduction of the electrical dimensions of the sections.

We attribute the observed improved TE-performance for sharp-edged dielectrics to the better characterization of the singular-fields thanks to the discontinuous transition of the expanded currents at corner-nodes and the convenient field testing inside the null-field regions of the associated homogeneous problems, especially over domains attached to corners, so that the null-field conditions assumed in the surface equivalence theorem are better enforced. Consistently, the nonconforming TM-analysis of dielectric objects or the nonconforming TE-analysis of smooth dielectric sections, with no singular field behavior, do not exhibit improved accuracy versus the number of unknowns when compared with the continuous analysis.

Our nonconforming EFIE-PMCHWT implementations are very useful in practice in the analysis of composite objects with piecewise homogeneous regions. The conventional node-based continuous schemes require an elaborate management of junction nodes, where several regions intersect. In contrast, the use of segment-based discontinuous basis functions in our nonconforming schemes, with no required knowledge about nodes over the boundary, circumvents the inconvenient management of junction nodes of the continuous schemes.

\section{REFERENCES}

[1] Roger F. Harrington, Field Computation by Moment Method, Macmillan, New York, 1968.

[2] S. M. Rao, D. R. Wilton and A. W. Glisson, "Electromagnetic scattering by surfaces of arbitrary shape," IEEE Transactions on Antennas and Propagation, vol. AP-30, No. 3, pp. 409-418, May 1982

[3] T. K. Wu and L. L. Tsai, "Scattering from arbitrarily-shaped lossy dielectric bodies of revolution,” Radio Sci., vol. 12, pp. 709-718, Sep.-Oct. 1977.

[4] Y. Chang and R. F. Harrington, "A surface formulation for characteristic modes of material bodies," IEEE Trans. Antennas Propag., vol. AP-25, pp. 789-795, Nov. 1977.
[5] A. J. Poggio and E. K. Miller, "Integral equation solutions of three-dimensional scattering problems," in Computer Techniques for Electromagnetics, R. Mittra, Ed. Oxford, U.K.: Pergamon, 1973, ch. 4.

[6] K. Umashankar, A. Taflove and S. M. Rao, "Electromagnetic scattering by arbitrary shaped three-dimensional homogeneous lossy dielectric objects," IEEE Transactions on Antennas and Propagation, Vol. AP-34, No. 6. June 1986.

[7] Roger F. Harrington, Time-harmonic Electromagnetic Fields, IEEE press, 2001.

[8] A. F. Peterson, S. L. Ray and R. Mittra, Computational Methods for Electromagnetics, IEEE Press, 1998.

[9] R. Hiptmair and C. Schwab, "Natural boundary element methods for the electric field integral equation on polyhedra," SIAM J. Numer. Anal., vol. 40, no. 1, pp. 66-86, 2002.

[10] A. Buffa, R. Hiptmair, T. von Petersdorff, and C. Schawb, "Boundary element methods for Maxwell transmission problems in Lipschitz domains," Numer. Math., vol. 95, pp. 459-485, 2003.

[11] P. Monk, Finite Element Methods for Maxwell's Equations, Oxford Science Publications, Clarendon Press, Oxford, 2003.

[12] Pasi Ylä -Oijala, Sami P. Kiminki, Johannes Markkanen, and Seppo Järvenpää, "Error-Controllable and Well-Conditioned MoM Solutions in Computational Electromagnetics: Ultimate Surface Integral-Equation Formulation," IEEE Antennas and Propagation Magazine, Vol. 55, No. 6, December 2013

[13] Pasi Ylä-Oijala, Sami P. Kiminki, and Seppo Järvenpää, "Conforming Testing of Electromagnetic Surface-Integral Equations for Penetrable Objects," IEEE Transactions on Antennas and Propagation, Vol. 64, No. 6, June 2016.

[14] K. Cools, F. P. Andriulli, D. De Zutter, and E. Michielssen, "Accurate and conforming mixed discretization of the MFIE," IEEE Antennas Wireless Propag. Lett., vol. 10, pp. 528-531, Jun. 2011

[15] E. Arvas, Y. Qian, T. K. Sarkar, and F. Alan, "TE scattering from a conducting cylinder of arbitrary cross-section covered by multiple layers of lossy dielectrics," Inst. Elec. Eng. Proc., vol. 136, pt H, pp. 425-430, 1989.

[16] Ahmed A. Kishk, Allen W. Glisson, and Paul M. Goggans, "Scattering from Conductors Coated with Materials of Arbitrary Thickness", IEEE Transactions on Antennas and Propagation, Vol. 40, No. 1, January 1992.

[17] M. Carr, E. Topsakal, and J. L. Volakis, "A procedure for modeling material junctions in 3-D surface integral equation approaches," IEEE Trans. Antennas Propag., vol. 52, pp. 1374-1379, May 2004.

[18] P. Ylä-Oijala, M. Taskinen, and J. Sarvas, "Surface integral equation method for general composite metallic and dielectric structures with junctions," Progr. Electromagn. Res., vol. 52, pp. 81-108, 2005.

[19] Branko M. Kolundzija, "Electromagnetic Modeling of Composite Metallic and Dielectric Structures," IEEE Transactions on Microwave Theory and Techniques, vol. 47, no. 7, July 1999.

[20] Yunhui Chu, Weng Cho Chew, Junsheng Zhao and Siyuan Chen, "A surface integral equation formulation for low-frequency scattering from a composite object", IEEE transactions on Antennas and propagation, vol. 51, no. 10, October 2003.

[21] I. Sekulic, E. Ubeda, J. M. Rius and A. Heldring, "Regularization of the 2D TE-EFIE for homogeneous objects discretized by the Method of Moments with discontinuous basis functions," International Conference on Electromagnetics in Advanced Applications, Aruba, Aug. 2014.

[22] I. Sekulic, E. Ubeda and J. M. Rius, "Tangential-normal line testing for a nonconforming discretization of the Transversal-Electric Electric-Field Integral Equation for 2D conductors," International Conference on Electromagnetics in Advanced Applications, Torino, Sep. 2015.

[23] E. Ubeda, Juan M. Rius and A. Heldring, "Nonconforming discretization of the Electric-Field Integral Equation for closed perfectly conducting objects," IEEE Trans. on Antennas Propagation, vol. 62, no. 8, Aug. 2014.

[24] E. Ubeda, J. M. Rius, A. Heldring and I. Sekulic, "Volumetric testing parallel to the boundary surface for a nonconforming discretization of the Electric-Field Integral Equation," IEEE Transactions on Antennas and Propagation, vol. 63, no. 7, pp. 3286-3291, July 2015.

[25] E. Ubeda, I. Sekulic, A. Heldring and J. M. Rius "Tangential-normal surface testing for the nonconforming discretization of the Electric-Field Integral Equation," IEEE Antennas and Wireless Propagation Letters, 2016.

[26] Lawrence F. Canino, John J. Ottusch, Mark A. Stalzer, John L. Visher, and Stephen M. Wandzura, "Numerical Solution of the Helmholtz Equation in 2D and 3D Using a High-Order Nyström Discretization," Journal of Computational Physics 146, 627-663, 1998.

[27] Andrew F. Peterson and Malcolm M. Bibby, An introduction to the Locally-Corrected Nystrom Method, Morgan \& Claypool Publishers, 2010.

[28] A. F. Peterson, "Accuracy of currents produced by the locally-corrected Nystrom method and the method of moments when used with higher order 
representations," Appl. Comput. Electromagn. Soc. J., vol. 17, no. 1, Mar. 2002.

[29] Aiming Zhu, Stephen D. Gedney and John L. Visher, "A study of combined field formulations for material scattering for a Locally Corrected Nyström discretization," IEEE Transactions on Antennas and Propagation, vol. 53 , no. 12 , pp. $4111-4120$, Dec. 2005.

[30] Nastaran Hendijani, Jin Cheng, Robert J. Adams, and John C. Young, "Constrained Locally Corrected Nyström Method," IEEE Transactions on Antennas and Propagation, Vol. 63, No. 7, July 2015.

[31] Z. Peng, K.H. Lim and J. F. Lee, "A discontinuos Galerkin surface integral equation method for electromagnetic wave scattering from nonpenetrable targets," IEEE Trans. Antennas Propagation, vol. 61, pp. 3617-3628, July 2013.

[32] Naveen V. Nair and Balasubramaniam Shanker, "Generalized method of moments: a framework for analyzing scattering from homogeneous dielectric bodies," J. Opt. Soc. Am. A, vol. 28, No. 3, March 2011.

[33] Mario A E. Bautista, F. Vipiana, M. A. Francavilla, J. A. Tobon Vasquez and G. Vecchi, "A nonconformal domain decomposition scheme for the analysis of multiscale structures," IEEE Trans. Antennas Propagation, vol. 63, pp. 3548-3560, August 2015.

[34] J. Van Bladel, Singular Electromagnetic Fields and Sources, Oxford Clarendon Press, 1991.

[35] I. Sekulic, E. Ubeda and J. M. Rius "Improved accuracy in the scattering analysis of infinitely long ferromagnetic objects", IEEE International Symposium on Antennas and Propagation \& USNC/URSI National Radio Science Meeting, Puerto Rico, June 2016.

[36] J. Meixner, "The behavior of electromagnetic fields at edges," IEEE Trans. Antennas Propagat., vol. AP-20, no. 4, pp. 442-446, July 1972.

[37] R. Mittra, and S. W. Lee, Analytical techniques in the theory of guided waves, New York: Macmillan, 1971.

[38] Budaev, B. V., and D. B. Bogy, "On the electromagnetic field singularities near the vertex of a dielectric wedge," Radio Sci., 42, RS6S08, 2007.

[39] M. M. Bibby, A. F. Peterson, and C. M. Coldwell "High-order representations for singular currents at corners," IEEE Trans. Antennas Propagat., vol. 56, n. 8, pp. 2277-2287, Aug. 2008.

[40] R. D. Graglia, and G. Lombardi, "Singular higher order complete vector bases for finite methods," IEEE Trans. Antennas Propagat., vol. 52, no.7, pp. $1672-1685$, July 2004.

[41] R. D. Graglia, and G. Lombardi, "Singular higher order divergence conforming bases of additive kind and moments method applications to 3D sharp-wedge structures," IEEE Trans. Antennas Propagat., vol. 56, no. 12, pp. 3768-3788, Dec. 2008

[42] G. Lombardi, and R. Graglia, "Modeling junctions in sharp edge conducting structures with higher order method of moments," IEEE Trans. Antennas Propagat., vol. 62, no. 11, Nov. 2014.

[43] L. N. Medgyesi-Mitschang, J. M. Putnam, and M. B. Gedera, "Generalized method of moments for three-dimensional penetrable scatterers", Journal of the Optical Society of America A, Vol. 11, No. 4, April 1994.

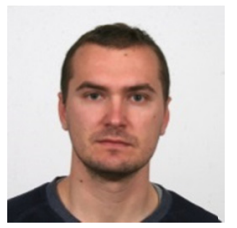

Ivan Sekulic was born in Pula, Croatia in 1987. He received the B.Sc. and M.Sc. degrees in electrical engineering and information technology from the Faculty of Electrical Engineering and Computing, University of Zagreb, Croatia, in 2008 and 2010, respectively. From 2011 to 2013 he worked at Koncar Electrical Engineering Institute, Zagreb, as Research Engineer. In 2013, he joined Department of Signal Theory and Communications (TSC), Universitat Politècnica de Catalunya (UPC), Barcelona, Spain as Research Assistant. His research interests include computational electromagnetics with focus on integral equation methods applied to the scattering analysis of composite structures.

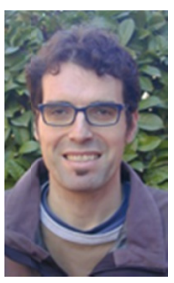

Eduard Ubeda was born in Barcelona, Spain, in 1971. He received the Telecommunication Engineer degree and the Doctor Ingeniero degree from the Politechnic University of Catalonia (UPC), Barcelona, Spain, in 1995 and 2001, respectively. In 1996, he was with the Joint Research Center, from the European Commission, in Ispra, Italy. From 1997 to 2000, he was a Research Assistant at the Electromagnetic and Photonic Engineering group, in the Department of Signal Theory and Communications (TSC), at UPC. From 2001 to 2002 he was a Visiting Scholar in the Electromagnetic Communication Laboratory of the Electrical Engineering Department at the Pennsylvania State University (PSU). Since 2003 he has been with the Electromagnetic and Photonic and Engineering Group, at UPC, where he currently holds the position of "Professor Agregat" (equivalent to Associate Professor). He is author of 28 papers in international journals and 70 in international conference proceedings. His main research interests are numerical computation of electromagnetic scattering and radiation using integral equations.

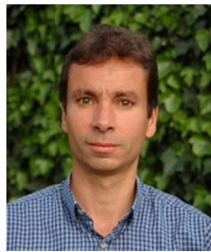

Juan M. Rius (S'88--M'91--SM'12) was born in Barcelona, Spain, in 1963. He received the "Ingeniero de Telecomunicación" degree in 1987 and the "Doctor Ingeniero" degree in 1991, both from the Universitat Politècnica de Catalunya (UPC), Barcelona, Spain. In 1985 he joined the Electromagnetic and Photonic Engineering group at UPC, Department of Signal Theory and Communications (TSC), where he currently holds a position of "Catedrático" (equivalent to Full Professor). From 1985 to 1988 he developed a new inverse scattering algorithm for microwave tomography in cylindrical geometry systems. Since 1989 he has been engaged in the research for new and efficient methods for numerical computation of electromagnetic scattering and radiation. He is the developer of the Graphical Electromagnetic Computation (GRECO) approach for high-frequency RCS computation, the Integral Equation formulation of the Measured Equation of Invariance (IE-MEI) and the Multilevel Matrix Decomposition Algorithm (MLMDA) in 3D. Current interests are the numerical simulation of electrically large antennas and scatterers. He has held positions of "Visiting Professor" at EPFL (Lausanne) from May 1, 1996 to October 31, 1996; "Visiting Fellow" at City University of Hong Kong from January 3, 1997 to February 4, 1997; "CLUSTER chair" at EPFL from December 1, 1997 to January 31, 1998; and "Visiting Professor" at EPFL from April 1, 2001 to June 30, 2001. He has more than 58 papers published or accepted in refereed international journals (34 in IEEE Trans.) and more than 159 in international conference proceedings. 